\title{
Design and Fabrication of Freeform Holographic Optical Elements
}

\author{
CHANGWON JANG, Facebook Reality Labs Research \\ OLIVIER MERCIER, Facebook Reality Labs Research \\ KISEUNG BANG, Facebook Reality Labs Research \\ GANG LI, Facebook Reality Labs Research \\ YANG ZHAO, Facebook Reality Labs Research \\ DOUGLAS LANMAN, Facebook Reality Labs Research
}
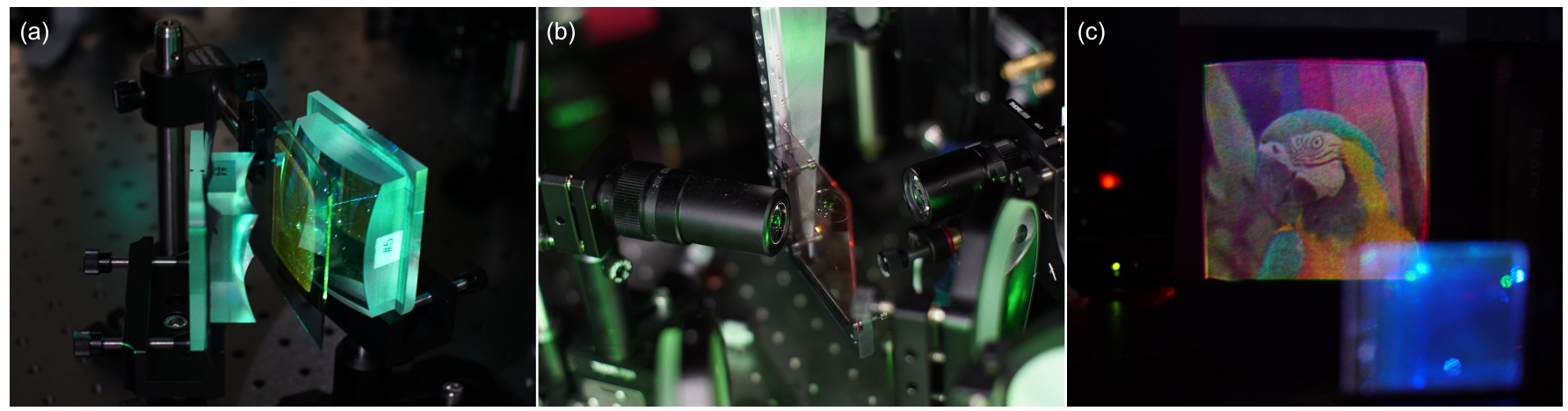

Fig. 1. We describe new approaches to design and fabricate freeform holographic optical elements (HOEs). Our unified optimization method for HOEs supports two means of fabrication: (a) using pairs of freeform refractive elements and (b) applying emerging holographic printing systems. (c) To showcase the capabilities of these design and fabrication methods, we demonstrate the first full-color caustic projection HOE. Parrot source image by Siripong Jitchum.

\begin{abstract}
Holographic optical elements (HOEs) have a wide range of applications, including their emerging use in virtual and augmented reality displays, but their design and fabrication have remained largely limited to configurations using simple wavefronts. In this paper, we present a pipeline for the design, optimization, and fabrication of complex, customized HOEs that enhances their imaging performance and enables new applications. In particular, we propose an optimization method for grating vector fields that accounts for the unique selectivity properties of HOEs. We further show how our pipeline can be applied to two distinct HOE fabrication methods. The first uses a pair of freeform refractive elements to manufacture HOEs with high optical quality and precision. The second uses a holographic printer with two wavefront-modulating arms, enabling rapid prototyping. We propose a unified wavefront decomposition framework suitable for both fabrication approaches. To demonstrate the versatility of these methods, we fabricate and characterize a series of specialized HOEs, including an aspheric lens, a head-up display lens, a lens array, and, for the first time, a full-color caustic projection element.
\end{abstract}

CCS Concepts: • Hardware $\rightarrow$ Emerging optical and photonic technologies; $\bullet$ Computing methodologies $\rightarrow$ Mixed / augmented reality.

Authors' addresses: Changwon Jang, Facebook Reality Labs Research; Olivier Mercier Facebook Reality Labs Research; Kiseung Bang, Facebook Reality Labs Research; Gang Li, Facebook Reality Labs Research; Yang Zhao, Facebook Reality Labs Research; Douglas Lanman, Facebook Reality Labs Research.

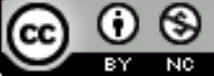

This work is licensed under a Creative Commons Attribution-NonCommercial International 4.0 License.

(c) 2020 Copyright held by the owner/author(s).

0730-0301/2020/12-ART184

https://doi.org/10.1145/3414685.3417762
Additional Key Words and Phrases: holography, holographic optical element, freeform optics

\section{ACM Reference Format:}

Changwon Jang, Olivier Mercier, Kiseung Bang, Gang Li, Yang Zhao, and Douglas Lanman. 2020. Design and Fabrication of Freeform Holographic Optical Elements. ACM Trans. Graph. 39, 6, Article 184 (December 2020), 15 pages. https://doi.org/10.1145/3414685.3417762

\section{INTRODUCTION}

Holographic optical elements (HOEs) are thin films typically up to tens of micrometers in thickness that interact with light through interference [Close 1975; Coufal et al. 2000; Kogelnik 1969]. In a typical HOE fabrication setup, a pair of coherent wavefronts (the signal and reference wavefronts) are interfered within a photosensitive material to create a volume grating. This volume grating may be described as a 3D vector field defined on the HOE surface, encapsulating the essential manner in which the HOE interacts with light. Illuminating a recorded HOE with the reference wavefront will output the original signal wavefront, thereby enabling the creation of thin optical elements with complex optical behaviors.

HOEs have been studied since the 1960s. Notably, in the 1990s and early 2000s, HOEs were considered for their potential use as a data storage medium. More recently, with the rising interest in virtual and augmented reality (VR/AR), research on HOEs is resurfacing due to their unique capabilities in shaping complex light wavefronts. HOEs avoid the strict physical limitations of conventional reflective and refractive optics that, for instance, constrain the maximum optical power of a lens based on its thickness and index 
of refraction (IOR). HOEs also possess useful properties originating from their volumetric grating structures, namely their selectivity and multiplexability. Selectivity is the ability to only diffract light from specific incoming angles or at specific wavelengths, which can be useful when designing VR/AR viewing optics [Koulieris et al 2019]. Multiplexability is the ability to superpose different configurations of volume gratings. This allows, for instance, an HOE to be recorded independently in red, green, and blue to create full-color images.

HOEs have been used for many computer graphics applications including head-mounted or near-eye displays [Akşit et al. 2019; Jang et al. 2017; Kim et al. 2019; Maimone et al. 2017], multi-layer displays [Lee et al. 2016a], head-up displays (HUDs) [Peng et al. 2014], or other imaging systems for holography or light fields [Haeussler et al. 2017; Jackin et al. 2018]. However, most prior applications only create HOEs using simple wavefronts, such as a planar or spherical wavefront with a specific direction or source points, which are relatively easy to record using traditional optics. For example, the HOE in a near-eye display mainly needs to reshape the spherical wavefront from a projector into a different spherical wavefront converging at the pupil of the user. In many cases, prior systems employing HOEs suffer from significant aberrations, limited diffraction efficiency, shrinkage of the material, or wavelength mismatches. As such, even though some efforts have been made to fine-tune HOE phase profiles [Amitai et al. 1990; Fairchild and Fienup 1982], there has been limited research into the design and optimization of complex, customized HOEs. Most previous works adopt 2D representations of HOEs without taking into account their angular and wavelength selectivity, and, therefore, do not enable their full design capabilities. For example, the selectivity properties of HOEs may be particularly useful to satisfy the challenging constraints on eye box, form factor, image resolution, and light efficiency required for $\mathrm{VR} / \mathrm{AR}$.

In this paper, we present a pipeline for the design and fabrication of freeform HOEs that can prescribe volume gratings with complex phase profiles and high selectivity. Our approach reduces image aberrations, optimizes the diffraction efficiency at a desired wavelength and angle, and compensates for the shrinkage of the material during HOE fabrication, all of which are highly beneficial for VR/AR applications. We also demonstrate the first full-color caustic HOE as an example of a complex, but smoothly-varying, volume grating.

We propose two different, but complementary, freeform HOE fabrication methods. The first uses diamond turning to create a pair of freeform refractive surfaces that shapes the wavefront for $\mathrm{HOE}$ recording. This method is slow and costly, but provides high optical quality and is suitable for industrial applications. The second uses a holographic printer to expose more degrees of freedom than prior fabrication methods. This approach has limited optical quality, but greatly accelerates fabrication, typically completing within only a few hours to support rapid iterations of emerging design concepts. Both fabrication methods require solving different optimization problems, and we provide algorithmic solutions in both cases, as well as demonstrate our solutions via experiments.

Our specific technical contributions include the following:
- an optimization method for freeform HOEs that accounts for their unique selectivity properties;

- two optimization methods for freeform HOE fabrication, including using diamond-turned freeform surfaces for highquality HOEs and a holographic printer with two wavefront modulating arms for rapid prototyping;

- robust wavefront decomposition algorithms tailored for both fabrication approaches;

- examples of display and imaging applications, including an AR image combiner, a head-up display, and a lens array; and

- the first demonstration of a full-color caustic projection HOE.

\section{RELATED WORK}

Freeform HOEs are closely related to the development of freeform lenses for imaging and illumination, as well as recent work in diffractive optical elements (DOEs) from the computational photography community. In this section we review prior work from these domains and provide an extended summary of HOEs for VR/AR.

\subsection{Freeform Lens Design for Imaging and Illumination}

When using traditional refractive optics for imaging systems, multiple simple lenses and optical elements can be combined for better performance. However, using fewer elements with more degrees of freedom in their optical surfaces is key to improving imaging performance and reducing the size of an optical stack. As such, several methods for designing freeform lenses have been widely applied [Bauer et al. 2018; Fang et al. 2013]. System configurations vary widely across imaging applications, with the corresponding freeform lens design methods typically tailored to specific design challenges. Many approaches adopt a form of multiple parameter optimization of the optical surfaces. Starting from a reasonable initial estimate, the space of possible surfaces is modeled using specific basis functions and the basis coefficients are optimized to improve some specific performance metric. This design approach is frequently used in industry [Garrard et al. 2005] since it can be adapted to specific configurations and it can optimize arbitrarily many degrees of freedom. Such methods are usually available in commercial tools like Code V [Synopsys 2020] or Optics Studio [Ze$\max 2020]$.

The design of illumination components is another application of freeform optics. In the computer graphics community, the design of freeform optical surfaces has been investigated for the generation of caustic patterns [Damberg and Heidrich 2015; Mérigot et al. 2017; Schwartzburg et al. 2014; Yue et al. 2014]. Caustics can be very complex and contain high-frequency features. As a result, it has proven difficult to optimize caustic surfaces using a decomposition into basis functions. Instead, the community has generally developed design methods relying on optimal mappings representing light transport.

\subsection{Holographic Optical Elements in VR/AR}

Various applications of HOEs have been studied for VR/AR. Peng et al. [2014] implemented a head-up display system using an offaxis lens HOE. Hong et al. [2014] implemented a 3D display by recording a lens-array $\mathrm{HOE}$, and a stack of HOEs has been used to 
implement multi-layer displays [Lee et al. 2016a]. HOEs are also part of many near-eye display systems, wherein HOEs are used as transparent reflective image combiners [Akşit et al. 2017; Jang et al. 2018, 2017; Kazempourradi et al. 2019; Kim et al. 2019; Maimone et al. 2017]. HOEs have also been used in place of waveguides for AR displays [Mukawa et al. 2008; Oku et al. 2015], as a thin VR display [Maimone and Wang 2020], and as an eye-tracking combiner element that is transparent to visible light, but sensitive to infrared illumination [Liu et al. 2018].

\subsection{Computational Photography}

Within the computational photography community, lithographybased diffractive optical elements (DOEs) have been used to overcome the limitations of conventional optical elements. By optimizing the point spread function (PSF) of DOEs and using deconvolution methods, recent works have demonstrated large depth of field and wide fields of view [Peng et al. 2019; Sitzmann et al. 2018]. Jeon et al. [2019] have also demonstrated hyperspectral imaging using DOEs with PSFs whose rotation encodes the hyperspectral information.

Similar to HOEs, DOEs have useful selectivity and multiplexability properties. The multiplexability of DOEs has been explored for computational photography by stacking multiple DOE layers [Ye et al. 2014], but this usually leads to significant noise, since stacked DOEs have low optical efficiency and low selectivity. Even though HOEs have better efficiency and selectivity than DOEs, there have been few projects in computational photography using HOEs. As a result, prior research into applying the multiplexability of DOEs is encouraging for the further development of complex HOE designs.

\subsection{Freeform HOEs and Holographic Printers}

Prior work on the design and fabrication of optimized HOEs has been relatively limited, since most efforts have focused on using simple spherical or planar wavefronts. In the 1970s, Fairchild and Fienup [1982] optimized the HOE phase for an aspheric design, which, to our knowledge, is the first mention of a freeform HOE design. However, the design and fabrication methods were limited by the computational capabilities and the fabrication tools at the time. They fabricated a computer-generated hologram and used a 4- $f$ optical relay system to filter the zero order component and high diffraction order noise, adding aberrations and limiting the numerical aperture (NA). Amitai et al. [1990] proposed using different wavelengths for the recording and reconstruction of HOEs to reduce aberrations, but their method does not generalize to complex designs. Crucially, these works do not take into account the selectivity of HOEs and rely on a 2D phase model for volume gratings, which cannot fully describe their 3D nature. Similar approaches are also used in more recent studies [Jackin et al. 2018; Jeong et al. 2019; Muslimov et al. 2018; Shen et al. 2013]. Many of the early HOE works use dichromated gelatin as a recording material, which yields large refractive index modulations. Modern applications now widely rely on photopolymers, which have smaller refractive index modulations that make selectivity a more important consideration. In this work, we show how the recording material can be taken into account during the volume grating optimization to incorporate the selectivity of the fabricated HOEs into their design.

Compared to the conventional HOE recording approach, holographic printers offer a more adaptable fabrication method where gratings are recorded as piecewise tiling patches called hogels. Such printers have been used before, for instance, to print holographic stereograms with 3D effects [Kim et al. 2015; Zebra Imaging 2020]. Wakunami et al. [2016] built holographic printers to fabricate HOE lenses for holographic HUDs, demonstrating that holographic printers could be a useful tool for imaging and display applications. Jeong et al. [2019; 2020] also used a holographic printer to design image combiners for near-eye displays. However, previous holographic printers only have a single wavefront modulator or spatial light modulator (SLM) arm, and a plane wave is used as the reference wave, which does not enable the complete design space for freeform HOEs. Recently, Hofmann et al. [2019] implemented a holographic printer with two modulation arms to fabricate infrared HOEs, but their applications are limited to wavelength-shifted recording, and they do not explore the optimization of freeform HOEs. In this paper, we demonstrate the implementation of a two-arm holographic printer combined with our optimization algorithm to achieve higher degrees of freedom for HOE fabrication.

\section{DESIGN METHOD}

This section presents our framework for modeling, optimizing, and fabricating freeform HOEs, as illustrated in Figure 2. We model volume gratings and wavefronts as $3 \mathrm{D}$ vector fields known as $k$ vectors [Close 1975; Kogelnik 1969; Wissmann et al. 2008], and use ray tracing to compute the interactions between light waves and the volume grating. This simple framework was first used by Close [1975] and has been used in multiple previous works [Fairchild and Fienup 1982; Jang et al. 2017; Wissmann et al. 2008]. Using this framework, we formulate the design and fabrication of HOEs as vector field construction and decomposition problems.

\section{$3.1 \quad k$-Vector Analysis}

Wavefronts are represented as 3D vector fields, where the norm of each vector, noted $k_{0}$, is

$$
k_{0}=2 \pi n_{h} / \lambda,
$$

where $n_{h}$ and $\lambda$ are, respectively, the refractive index and the wavelength of light inside the HOE medium. The direction of a vector is the local gradient of the wavefront (in an isotropic medium). This model enables us to treat the local wavefront as a ray when describing the interaction of wavefronts with the HOE. Since the HOE is very thin, we assume the volume grating can be represented as a $3 \mathrm{D}$ vector field lying on a $2 \mathrm{D}$ domain. With this framework, the grating vector $\overrightarrow{K_{G}}$ resulting from the interference of a reference wavefront $\overrightarrow{K_{R}}$ and a signal wavefront $\overrightarrow{K_{S}}$ is simply computed as $\overrightarrow{K_{G}}(x, y)=\overrightarrow{K_{G}}=\overrightarrow{K_{S}}-\overrightarrow{K_{R}} \in \mathbb{R}^{3}$.

We denote the grating vector as $\overrightarrow{K_{G}}=\left(K_{G, x}, K_{G, y}, K_{G, z}\right)$. The magnitude of each component is inversely proportional to the period $\Lambda$ of the volumetric grating in each axis direction, so $\left|K_{G, x}\right|=2 \pi / \Lambda_{x}$, and similarly for $y$ and $z$. For convenience, the $k$-vectors are defined inside the recording volume of the HOE, and Snell's law must be 

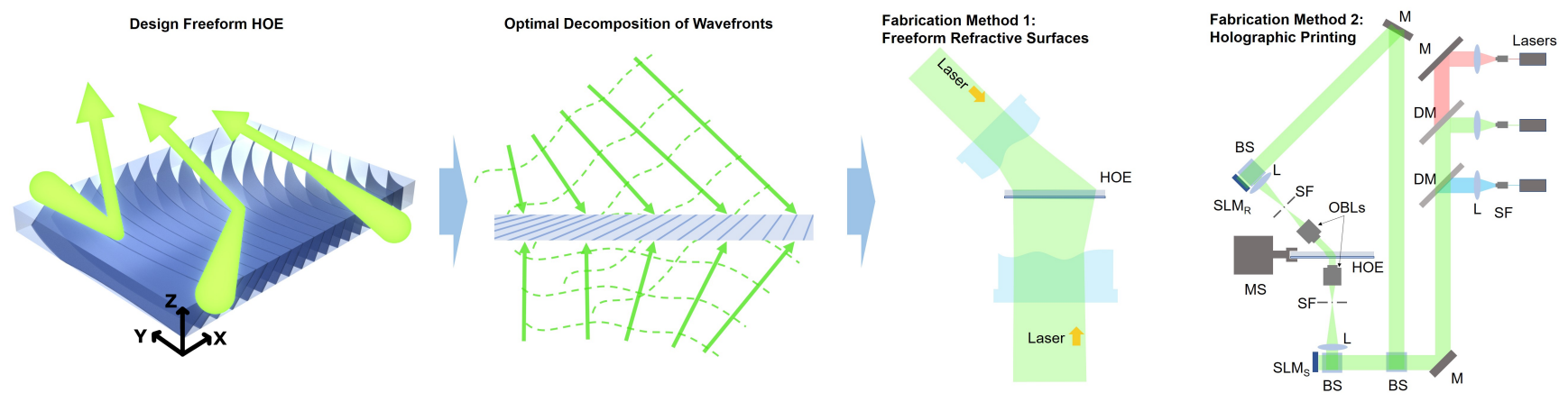

Fig. 2. Overview of our freeform HOE fabrication pipeline. The refractive index modulation in a holographic material forms a volume grating, and we refer to an HOE with a spatially-varying pitch and slant angle as a freeform HOE. First, we design the volume grating vector field of the freeform HOE (left). The slant angles of the grating are exaggerated here for visualization. Second, we decompose the grating as two interfering wavefronts (middle-left). These wavefronts are optimized depending on the fabrication method: the first method (middle-right) uses a pair of diamond-turned freeform refractive surfaces. The second method (right) uses a holographic printer with two modulation arms, which can print the hologram as a piecewise collection of tiling patches called hogels. The figure labels indicate the spatial filters (SF), lenses (L), mirrors (M), dichroic mirrors (DM), objective lenses (OBL), mechanical stage (MS), reference and signal spatial light modulators $\left(\mathrm{SLM}_{R / S}\right)$, and the beam splitters (BS). See Section 4.4 for details.

applied to refract an incoming wavefront before it interacts with the HOE. When a ray with $k$-vector $\overrightarrow{K_{\text {in }}}$ hits the volume grating $\overrightarrow{K_{G}}$, the first-order diffracted light gives the simple relation [Saleh and Teich 2007]

$$
\left(K_{\text {out }, x}, K_{\text {out }, y}\right)=\left(K_{\mathrm{in}, x}+K_{G, x}, K_{\mathrm{in}, y}+K_{G, y}\right),
$$

where $\overrightarrow{K_{\text {out }}}$ is the resulting outgoing ray. Since $\left\|\overrightarrow{K_{\text {out }}}\right\|=k_{0}$, Equation 2 also determines $K_{\mathrm{out}, z}$, so the $x$ and $y$ components of the grating completely determine the output direction of $\overrightarrow{K_{\text {out }}}$. The $z$ component of the grating still has an important effect on the selectivity of the HOE: when light interacts with the volume grating, only a portion of the incoming light is diffracted, while the rest is mostly transmitted. We define the phase mismatch $\triangle K$ as

$$
\triangle K=K_{\text {out }, z}-K_{\mathrm{in}, z}-K_{G, z},
$$

and the efficiency of the HOE diffraction is strongest when $\triangle K=0$, which is known as the Bragg diffraction condition. The general goal of HOE design is thus to find $\overrightarrow{K_{G}}$ that satisfies Equation 2 for a given set of input and output $k$-vector pairs at a given wavelength, while minimizing $\Delta K$. Note that these conditions can usually not all be satisfied exactly, and the problem must be regularized (e.g., by solving it in the least-squares sense).

\subsection{Freeform HOE Optimization}

To help align the grating to the geometry of the recording setup, we divide the grating components into a fixed initial grating $\overrightarrow{K_{G 0}}(x, y)$ and a freeform term $\overrightarrow{d K_{G}}(x, y)$, to get

$$
\overrightarrow{K_{G}}(x, y)=\overrightarrow{K_{G 0}}(x, y)+\overrightarrow{d K_{G}}(x, y) .
$$

The optimization starts from the initial grating specified by the system configuration (e.g., reflection or transmission system, axis alignment, converging or diverging optical power) and gradually modifies the freeform term to find the optimal $k$-vector design. We adopt a multi-parameter optimization approach, and decompose the freeform term into a linear combination of basis functions independently for each component:

$$
\overrightarrow{d K_{G}}(x, y)=\left(\sum_{n} a_{x n} b_{x n}, \sum_{n} a_{y n} b_{y n}, \sum_{n} a_{z n} b_{z n}\right),
$$

where $a_{\cdot n}$ are coefficients and $b_{\cdot n}=b_{\cdot n}(x, y)$ are scalar basis functions. Various representations (e.g., Zernike and Fourier) can be used as basis functions. Since the applications in this paper mainly concern HOEs with rectangular apertures, we use gradients of Legendre polynomials for $b_{x n}, b_{y n}$, and Legendre polynomials for $b_{z n}$ up to sixth order, as detailed in our supplementary document (Section 1.2). This yields satisfactory results for imaging applications since the HOE profiles tend to be smooth, but the number of basis functions can be increased as high as desired for complex profiles.

From Equations 2 and 3, we take advantage of the fact that $\overrightarrow{K_{\text {out }}}$ is solely determined by $K_{G, x}$ and $K_{G, y}$ to separate the optimization process into a point spread function (PSF) pass on the $K_{G, x}$ and $K_{G, y}$ components and a selectivity pass on the $K_{G, z}$ component. We use a ray tracing simulation to evaluate the performance of the HOE during the optimization process. This process is visualized in Figure 3, where we jointly optimize two HOEs to produce an imaging optical element between a source plane and a sensor.

PSF optimization. We first optimize the PSF of the imaging system by manipulating $a_{x n}$ and $a_{y n}$. Starting on the aperture of the sensor, bundles of rays are traced at different viewing angles toward the source plane, where we minimize the PSF of each bundle as

$$
\left\{a_{x n}, a_{y n}\right\}=\underset{\left\{\widehat{a}_{x n}, \widehat{a}_{y n}\right\}}{\operatorname{argmin}} \sum_{j}^{\text {bundles rays }} \sum_{i}\left\|\overline{p_{j}}-p_{i, j}\right\|^{2}+\sum_{j}^{\text {bundles rays }} \sum_{i} \Omega(i, j),
$$

where $p_{i, j}, \overline{p_{j}}$, and $\Omega_{i, j}$ depend implicitly on basis coefficients $\widehat{a}_{x n}$ and $\widehat{a}_{y n}$. Namely, $p_{i, j}$ is the position of ray $i$ of bundle $j$ at the source plane, $\overline{p_{j}}$ is the average location of rays in bundle $j$, and $\Omega_{i, j}$ is a penalty term to satisfy boundary constraints, i.e., to avoid total internal reflection of the rays and to keep the rays within the 

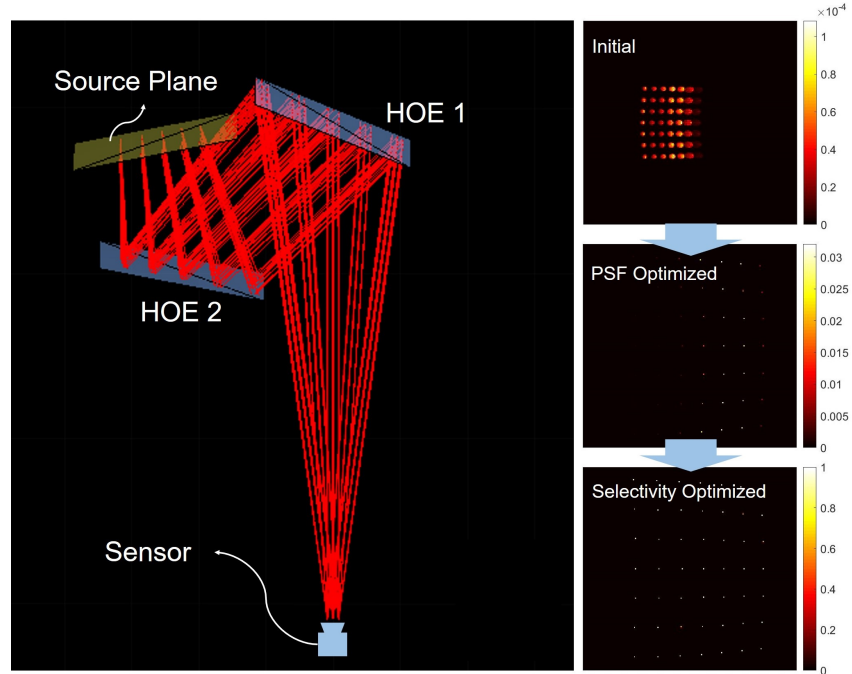

Fig. 3. Illustration of the raytracing configuration and optimization process. We separately optimize the PSF and the selectivity of the HOE. Before the optimization, the PSF has severe aberrations and its diffraction efficiency is very low because of the shrinkage of both HOEs (top-right). After PSF optimization, the ray bundles are sharply focused at the source plane, but the efficiency is still low (middle-right). After selectivity optimization, both HOEs are tuned to have maximum and uniform efficiency in the desired configuration (bottom-right). Colorbars show the relative intensity normalized to maximum. We also illustrate this process in our supplementary video.

aperture of each element. We use the interior point method [Byrd et al. 2000] to solve this minimization, which gives us the coefficients $\left\{a_{x n}\right\}$ and $\left\{a_{y n}\right\}$ of the optimized gratings. The $\left\{a_{z n}\right\}$ coefficients are not determined during this pass, since $K_{G, z}$ does not affect the output direction of rays.

Selectivity optimization. The selectivity of the HOE is a function of HOE material characteristics such as its thickness and refractive index modulation, as well as the phase mismatch $\Delta K_{z}$ specified by Equation 3. We optimize the coefficients $a_{z n}$ to maximize the diffraction efficiency (DE) as

$$
\left\{a_{z n}\right\}=\underset{\left\{\widehat{a}_{z n}\right\}}{\operatorname{argmin}} \sum_{j}^{\text {bundles rays }} \sum_{i}-S(i, j) I_{i, j},
$$

where $S$ and $I_{i, j}$ depend implicitly on basis coefficients $\widehat{a}_{z n}$. Namely, $S$ is a selection function that weighs contributions according to the incidence angle or wavelength of each ray, and $I_{i, j}$ is the intensity of the ray that is modulated due to the selectivity of HOE. The cost function can be either designed to optimize the HOE to yield maximum diffraction efficiency (DE) values, or to tune it to some target efficiency. We adopt the coupled wave theory (CWT) diffraction efficiency model of Yariv and Yeh [1983], which is known to show a good match with rigorous simulation results. However, we found that this optimization can sometime stay in local minima since this model has side lobes in its selectivity equation. Therefore we propose to approximate the selectivity function with a polynomial for apodization as

$$
\eta_{\text {approx }}=\tanh \left(\frac{2 \pi n_{d} k_{0} t}{\lambda\left\|\overrightarrow{K_{G}}\right\|}\right)^{2} \frac{1}{1+\left(\frac{t \Delta K}{2 \pi}\right)^{4}},
$$

where $n_{d}$ is the amplitude of refractive index modulation of the volume grating and $t$ is the thickness of the volume hologram material. For more details about this diffraction efficiency function and our modeling, see our supplementary document (Section 1.1).

Note that we optimize the HOE designs for a fixed configuration, meaning the wavelength, refractive index, and propagation distance are fixed. However, these parameters could easily be added to our formulation in Equations 6 and 7 as additional optimization parameters, but we chose to restrict our optimization only to the k-vectors of the HOE to simplify the problem description.

Design example. Based on the modeling and optimization process above, we demonstrate a design example of an off-axis head-up display (HUD) system. The off-axis configuration is attractive for designing the optical path and form factor of the system; however, it usually induces severe aberrations that degrade the image quality [Jang et al. 2017; Lee et al. 2016b; Peng et al. 2014]. We use this configuration to design two systems: a singlet HUD system with a single HOE, and a doublet HUD system with two reflective HOEs to reduce aberration and maximize the diffraction efficiency. Figure 10 shows the configuration of these HOEs, and more details are given in our supplementary document (Section 2.1).

To verify our design, we assess the resolution of the designed example using Code V [Synopsys 2020] by comparing our freeform HOEs with a baseline hologram and with previous work. We show simulation results in Figure 4. The baseline HOE is reconstructed from a basic spherical wave and a plane wave, which has diffractionlimited resolution at the center of the field of view; however, it has severe aberrations in other fields. We also compare with the work of Lee et al. [2016b], which corrects the aberrations by recording HOEs with cylindrical lens profiles. In comparison, our designs show noticeably better imaging quality over the field of view. The efficiency is not considered here since Code $\mathrm{V}$ does not support the simulation of efficiency for HOEs, but we provide experimental results that show the efficiency improvements in Figure 12. Note that we do not directly compare with the design of Peng et al. [2014] since our specifications are different, but since we achieve an almostdiffraction-limited resolution over the entire field of view, our design is comparable to theirs. Considering that Peng et al. use ten optical elements in total for correcting these aberrations, this shows that our freeform HOE optimization can be a simpler and more powerful tool for VR/AR applications.

In Figure 10, we present other design examples with configurations including aberration-corrected and selectivity-optimized on-axis birdbath-type AR combiner lens (aspheric lens), an off-axis HUD screen (HUD singlet), and a doublet version of the HUD lens (HUD doublet). In addition, as a more challenging design, we create a lens array HOE (off-axis lens array) which can be used for an offaxis multi-view eye-tracking element, where lenslets are optimized separately and stitched together. Details of each configuration are provided in our supplementary document (Section 2.1). 

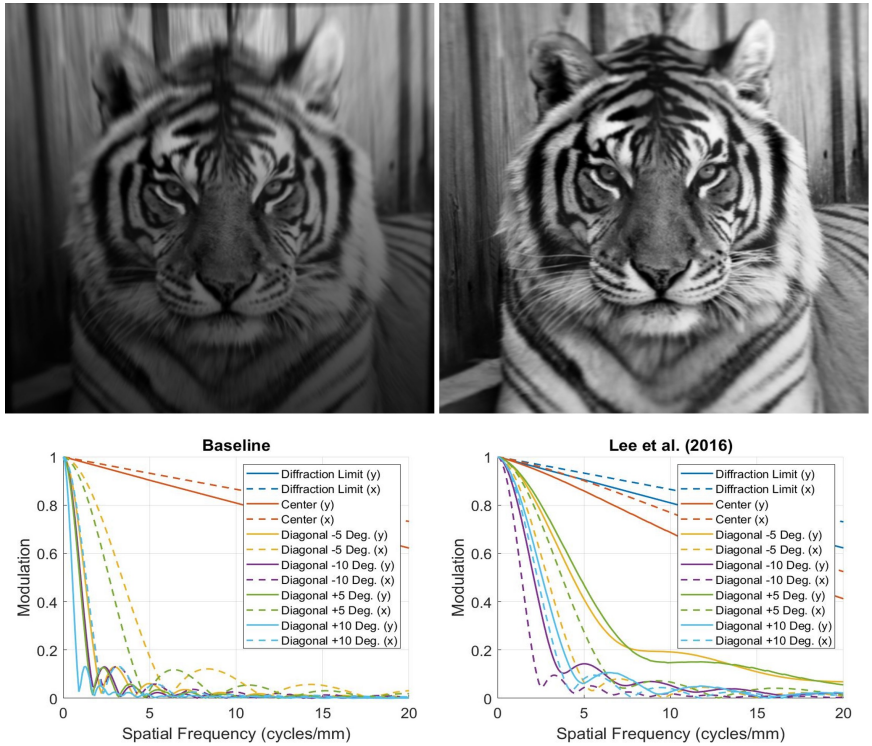

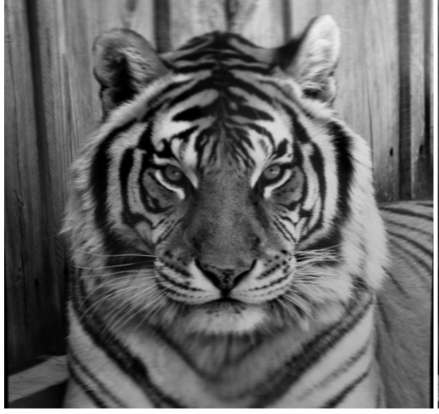

Ours (singlet)

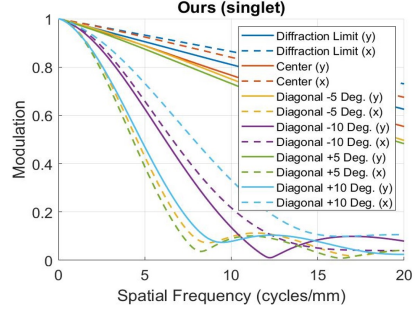

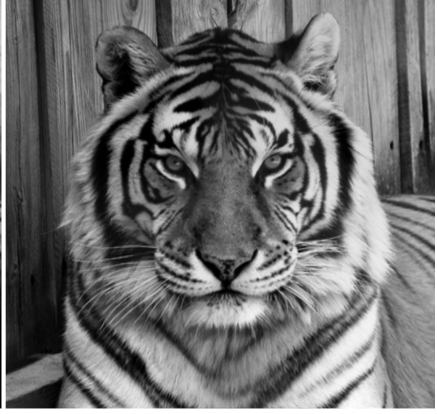

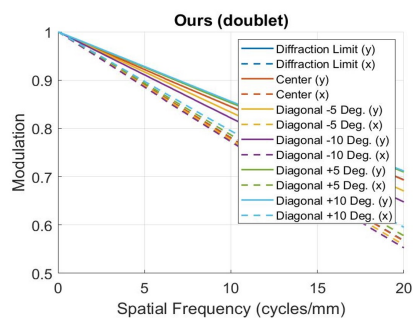

Fig. 4. Resolution comparison of simulated imaging systems using, from left to right, a baseline lens HOE, an astigmatism-corrected HOE lens [Lee et al. 2016b], our singlet freeform HOE, and our doublet freeform HOEs. The modulation transfer function of each HOE design is shown in the bottom row. Our freeform HOEs generate better image resolutions compared to the baseline lens HOEs or previous work, especially for the doublet HOE design which shows a resolution close to the diffraction limit of a $3 \mathrm{~mm}$ pupil. Images have been undistorted to facilitate comparisons. Tiger source image by LMPphoto.

Shrinkage compensation. Typical materials (e.g., photopolymers) show shrinkage in the $z$-axis after curing, while shrinkage on the lateral axes is negligible. This shrinkage is a material characteristic and is reproducible across samples. This $z$-axis shrinkage shifts the maximum diffraction efficiency angle or wavelength by a few degrees or a few nanometers, which results in significant efficiency drops or undesired aberrations. There have been several attempts to compensate for shrinkage by changing the recording configuration [Fang et al. 2013]. For instance, when recording simple plane wave gratings, the shrinkage can be compensated by tilting the recording angle. However, when the volume grating vector varies over the area, for example in a lens HOE, it cannot be compensated in this simple manner. A compensation method has been proposed using different recording and reconstruction wavelengths [Amitai et al. 1990], but this method is limited to specific designs and cannot be applied to HOEs having complex profiles. Instead, we propose to pre-compensate for the shrinkage by re-scaling the $z$ component as

$$
{\overrightarrow{K_{G}}}^{\prime}=\left(K_{G, x}, K_{G, y},(1-\alpha) K_{G, z}\right),
$$

where $\alpha$ is the material shrinkage coefficient. This compensation method can be applied to any complex freeform grating profile since it simply changes the parameterized vector field.

\subsection{Caustic HOE Optimization}

Caustics are light projections caused by irregularly-shaped refractive or reflective surfaces. The design of optical elements to create specific caustics is an interesting extension of conventional freeform illumination design that has been investigated in the computer graphics community [Damberg and Heidrich 2015; Mérigot et al
2017; Schwartzburg et al. 2014; Yue et al. 2014]. Some computergenerated holograms can also create images by simulating wave propagation directly to fabricate the proper phase masks [Peng et al. 2017]. However our caustic HOEs are distinct from computergenerated holograms since ours are based on smooth optical power variations rather than complicated diffraction phase patterns. To show the versatility of our HOE optimization pipeline, we demonstrate, for the first time, the construction of a full-color caustic HOE.

While the design of imaging HOEs usually works with simple, smooth profiles, an impractically large number of basis is required to create natural images with high-frequency details for caustic illumination. Instead, we decompose the problem with a much more tractable method. First, we compute an optimal light transport mapping $T:(x, y, z) \mapsto\left(x_{t}, y_{t}, z_{t}\right)$ from the HOE plane to the target field by solving a Poisson equation [Yue et al. 2014]. This indicates how the light at the glass surface should be gathered on the target surface to generate the desired caustic. We use the caustic source illumination wavefront $\overrightarrow{K_{\text {illum }}}(x, y)$ as our reference wavefront. The mapping is then converted to the target grating vector field as a difference between the light transporting wavefront $\overrightarrow{K_{T}}$ and the illumination wavefront as

$$
\overrightarrow{K_{g}}(x, y)=\left(K_{T, x}, K_{T, y}, \sqrt{\frac{2 \pi n_{h}^{2}}{\lambda}-K_{T, x}^{2}-K_{T, y}^{2}}\right)-\overrightarrow{K_{\mathrm{illum}}}
$$

where

$$
\overrightarrow{K_{T}}=\frac{2 \pi}{\lambda} \frac{\left(x_{t}, y_{t}, z_{t}\right)-(x, y, z)}{\left\|\left(x_{t}, y_{t}, z_{t}\right)-(x, y, z)\right\|}
$$

Compared to refractive caustic lenses, our caustic HOE is a flat film that can provide the required optical power without the thickness 
constraints of a glass element. The selectivity and multiplexability properties of the $\mathrm{HOE}$ are also key to generating completely independent caustics in each color channel, which is not possible with refractive optics. We have created a full-color caustic HOE, as shown in Figure 11 (b), and demonstrate the results in Figures 12.

\section{FABRICATION METHOD}

\subsection{Optimal Vector Field Decomposition}

Once the grating vector field $\overrightarrow{K_{G}}$ has been designed, we need to find a pair of signal and reference wavefronts to fabricate the freeform HOE. In the fabrication stage, the grating vector is formed as the difference of the $k$-vectors of the signal and reference beams. Therefore, fabrication is essentially a vector field decomposition problem to find $\overrightarrow{K_{S}}$ and $\overrightarrow{K_{R}}$ such that

$$
\overrightarrow{K_{G}}(x, y)=\overrightarrow{K_{S}}(x, y)-\overrightarrow{K_{R}}(x, y),
$$

subject to

$$
\left\|\overrightarrow{K_{S}}(x, y)\right\|=\left\|\overrightarrow{K_{R}}(x, y)\right\|=k_{0}
$$

and

$$
\begin{aligned}
& \left|K_{S, z}(x, y)\right|>k_{0} \sin \left(n_{s} / n_{h}\right), \\
& \left|K_{R, z}(x, y)\right|>k_{0} \sin \left(n_{s} / n_{h}\right),
\end{aligned}
$$

where $n_{h}$ and $n_{s}$ are, respectively, the refractive indices of the HOE material and the surrounding media (usually air). Equation 14 prevents total internal reflection inside the HOE.

This decomposition is under-determined and there exists an infinite number of solutions (provided that $\left\|\overrightarrow{K_{G}}\right\|<2 k_{0}$ ). As illustrated in Figure 5, there is a 1D set of vector pairs that satisfies Equations 12 and 13 for a given $\overrightarrow{K_{G}}$. We use $\theta$ to parameterize these decomposition solutions as

$$
\begin{aligned}
& \overrightarrow{K_{S}}(\theta)=\frac{\overrightarrow{K_{G}}}{2}+\sqrt{k_{0}^{2}-\left\|\overrightarrow{\frac{K_{G}}{2}}\right\|^{2}}(\vec{x} \cos (\theta)+\vec{y} \sin (\theta)), \\
& \overrightarrow{K_{R}}(\theta)=-\frac{\overrightarrow{K_{G}}}{2}+\sqrt{k_{0}^{2}-\left\|\overrightarrow{\frac{K_{G}}{2}}\right\|^{2}}(\vec{x} \cos (\theta)+\vec{y} \sin (\theta)),
\end{aligned}
$$

where

$$
\begin{gathered}
\vec{y}=\frac{\overrightarrow{K_{G}} \times(1,0,0)}{\left\|\overrightarrow{K_{G}} \times(1,0,0)\right\|}, \\
\vec{x}=\frac{\vec{y} \times \overrightarrow{K_{G}}}{\left\|\vec{y} \times \overrightarrow{K_{G}}\right\|},
\end{gathered}
$$

and $\times$ denotes the cross product. Note that for any practical situation, $\overrightarrow{K_{G}}$ has small $x$ and $y$ components, which prevents singularities in Equations 17 and 18.

The decomposed $\overrightarrow{K_{S}}$ and $\overrightarrow{K_{R}}$ give the direction of a light ray at each position on the HOE, and the $k$-vectors can be converted to wavefronts by integration. Note that this decomposition warrants the modulation of both the signal and reference wavefront. If either wavefront is fixed, $\overrightarrow{K_{S}}$ and $\overrightarrow{K_{R}}$ cannot always lie on the solution space and therefore cannot be fabricated exactly. In most previous works, the reference wavefront is fixed and predetermined by the
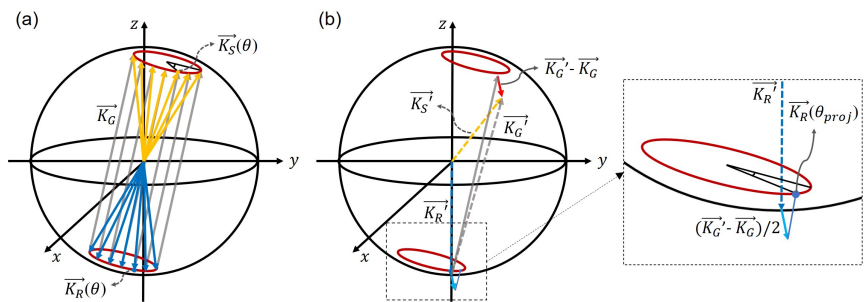

Fig. 5. Parameterization of the vector field decomposition. (a) Yellow arrows and blue arrows indicate possible solutions for $\overrightarrow{K_{S}}$ and $\overrightarrow{K_{R}}$, respectively, and the grating vector $\overrightarrow{K_{G}}$ is indicated with gray arrows. The red circles show the solution space of the parameterization for $\overrightarrow{K_{S}}$ and $\overrightarrow{K_{R}}$. (b) Current decomposition vector pairs are indicated as dotted arrows. Updated decomposition pairs are computed by projecting the sum of $\overrightarrow{K_{R}^{\prime}}$ and $\left(\overrightarrow{K_{G}^{\prime}}-\overrightarrow{K_{G}}\right) / 2$ to the solution space.

recording setup, and there is no guarantee that a corresponding signal wavefront can be found to satisfy Equations 12-14, which leads to approximate solutions.

In comparison, we propose two different fabrication methods that modulate both wavefronts, as shown in Figure 2, so we have full access to this solution space and thus we can use it for further optimization. Out first method uses a pair of freeform refractive elements to generate the target wavefronts. Our second method uses a holographic printer with two wavefront modulation arms that discretizes the freeform HOE as hogel patches. Depending on the fabrication method, the wavefronts may need to satisfy additional constraints. We optimize the remaining degree of freedom $\theta$ to better satisfy the constraints of each fabrication method, as detailed in the following two sub-sections. We refer to our optimized values for $\theta$ at each point $(x, y)$ of the HOE as the $\theta$ map.

\subsection{Freeform Surface Optimization}

One way to generate freeform wavefronts $\overrightarrow{K_{S}}$ and $\overrightarrow{K_{R}}$ is to use a simple illumination source (e.g., collimated rays) refracted through a freeform element (usually made of glass). The forward propagation of rays through glass is a simple application of Snell's law, but the converse problem of generating a glass surface that generates a target wavefront is not trivial and a solution might not always exist.

In most previous works [Damberg and Heidrich 2015; Mérigot et al. 2017; Schwartzburg et al. 2014; Yue et al. 2014], both wavefronts are fixed and must be regularized so they can be approximately generated by a glass surface. In our approach, since we do not determine any of the recording wavefronts a priori, we can compute the refractive surface and the wavefront conjointly. We use the additional degree of freedom given by the $\theta$ map to compute wavefronts that are as close as possible to wavefronts that can be generated by refractive elements, which can yield more accurate solutions. However, because refraction laws depend non-linearly on surface normals, and the $K_{z}$ component of a wavefront depends non-linearly on the $K_{x}$ and $K_{y}$ components, it is not simple to formulate a minimization metric in order to compute the optimal $\theta$ map, so we instead use a more direct iterative approach. 


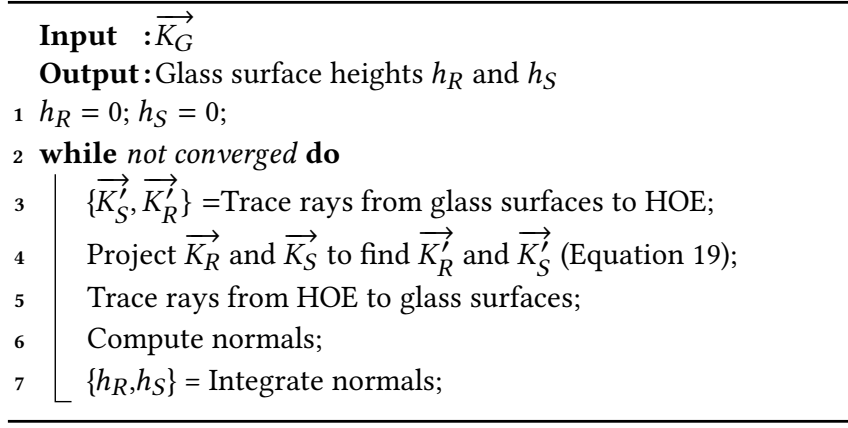

Algorithm 1: The glass optimization method of Section 4.2.

Our method is summarized in Algorithm 1 and illustrated in our supplementary video. Starting from flat glass surfaces, we trace rays from the illumination sources to the HOE surface for both the reference and signal paths. The rays are collected at the HOE surface, which gives temporary wavefronts $\overrightarrow{K_{S}^{\prime}}$ and $\overrightarrow{K_{R}^{\prime}}$. Since $\overrightarrow{K_{S}^{\prime}}-\overrightarrow{K_{R}^{\prime}} \neq \overrightarrow{K_{G}}$ at this stage, we project $\overrightarrow{K_{S}^{\prime}}$ and $\overrightarrow{K_{R}^{\prime}}$ to the solution space in order to recover $\overrightarrow{K_{G}}$ exactly at the surface. To apply this projection, we first compute $\theta_{\text {proj }}$ by solving

$$
\begin{aligned}
& \cos \left(\theta_{\text {proj }}\right)=\left(\frac{\overrightarrow{K_{S}^{\prime}}+\overrightarrow{K_{R}^{\prime}}}{2}\right) \cdot \vec{x} \\
& \sin \left(\theta_{\text {proj }}\right)=\left(\frac{\overrightarrow{K_{S}^{\prime}}+\overrightarrow{K_{R}^{\prime}}}{2}\right) \cdot \vec{y},
\end{aligned}
$$

where $\vec{x}$ and $\vec{y}$ use the definitions of Equations 17 and 18. We then use $\theta_{\text {proj }}$ in Equations 15 and 16 to compute the projected $\overrightarrow{K_{S}}$ and $\overrightarrow{K_{R}}$. This projection satisfies $\overrightarrow{K_{S}}-\overrightarrow{K_{R}}=\overrightarrow{K_{G}}$ exactly, without moving too far away from $\overrightarrow{K_{S}^{\prime}}$ and $\overrightarrow{K_{R}^{\prime}}$.

The projected $\overrightarrow{K_{S}}$ and $\overrightarrow{K_{R}}$ are then ray-traced backward from the HOE to the glass surface. At each intersection point on the glass surface, we compute the surface normal required for the glass to refract the illumination ray to the $\overrightarrow{K_{S}}$ or $\overrightarrow{K_{R}}$ direction. Finally, the refractive surface is reconstructed from its normals, similarly to the method of Harker and O'Leary [2008]. This gives a new glass surface, and the process is repeated until convergence. It is convenient to have continuously varying normals on the glass surface for accurate refraction computations, so we use cubic interpolants defined on a grid to represent the surface profiles. We also ignore the total internal reflection conditions of Equation 14 in our algorithm, since, in practice, the surfaces are smooth enough that total internal reflection does not occur during optimization.

Note that the surface reconstruction step is not exact, since the set of normals will, in general, not be integrable to a surface. Also, since the refracted rays depend on both the surface normal and surface height and since the reconstruction step changes the surface height, the refracted wavefront will not be exactly reconstructed, even if the normals are perfectly integrable. Nevertheless, this projection approach converges, in practice, to glass surfaces that can accurately generate the desired grating vectors. This simple ray-tracing framework is also easily expandable to other recording configurations, for instance using a combination of fixed and freeform optical elements, or working in an immersed configuration.

Figure 6 shows the evolution of the surfaces for an aspheric lens HOE (top), as well as the errors for multiple HOE designs (bottom right). The error in this figure is defined as

$$
\text { error }=\operatorname{RMS}\left(\frac{\left\|\overrightarrow{K_{G}}-\overrightarrow{K_{G}^{\prime}}\right\|}{\left\|\overrightarrow{K_{G}}\right\|}\right) \text {, }
$$

where RMS gives the root mean square, and $K_{G}^{\prime}=\overrightarrow{K_{S}^{\prime}}-\overrightarrow{K_{R}^{\prime}}$. The recording configurations and final surfaces for each $\mathrm{HOE}$ are also shown in Figure 10. For each HOE example except the lens array, our method converges nicely to a solution with an error of at most $10^{-4}$. For reference, an error of $10^{-3}$ provides a diffraction error of 0.18 degrees in the worst case, and an error of $10^{-4}$ yields about 1 arcminute of diffraction error, which is below the acuity limit of normal human vision.

The bottom left plot of Figure 6 compares our method to the case where one of the wavefronts is fixed a priori, similarly to what is done in previous studies of caustic lens optimization [Damberg and Heidrich 2015; Mérigot et al. 2017; Schwartzburg et al. 2014; Yue et al. 2014]. This greatly simplifies the iterative process, since the constant reference wavefront fixes the $\theta$ map in place, and only the surface reconstruction part of the method needs to be computed. Although this method converges faster (in only 2 or 3 iterations), our method is able to converge to glass surfaces that yield errors almost 4 orders of magnitude smaller in some cases.

Note that for the Parrot Caustic example, the error isn't as stable as in the other cases. This caustic contains high frequency image details, which we believe challenges the convergence. Still, this is not an issue for fabrication, since the error converges and is small enough to be imperceptible, but this behavior warrants further investigation. The Lens Array example is a very extreme and discontinuous case, but our method still manages to converge to a smooth solution. A piecewise computation might be better suited for this scenario, since the error only reaches $10^{-2}$ and is dominated by the smoothed discontinuities, but this test confirms the robustness of our method.

\subsection{Fabrication of Freeform Surfaces}

The optimized surfaces are converted to $3 \mathrm{D}$ models and roughly milled using a CNC machine. Each surface is then refined using a diamond turning machine, as shown in Figure 11 (a). Diamond turning has a precision of several nanometers, which is sufficient to generate surfaces of optical quality without additional finishing. This approach imposes an additional constraint on the surfaces, since the diamond turning machine limits the slope of the surface along the tangential direction, in our case to 20 degrees. However, in practice, the maximum angle of our optimized surfaces is 17 degrees, so the constraint is naturally satisfied. We fabricated the refractive elements using PMMA as our material and recorded the HOEs for an on-axis aspherical lens, an off-axis HUD, a caustic HOE in green only (532 nm, IOR 1.49), and a full-color caustic parrot HOE (red $660 \mathrm{~nm}$, IOR 1.485, and blue $473 \mathrm{~nm}$, IOR 1.495) using this diamond 

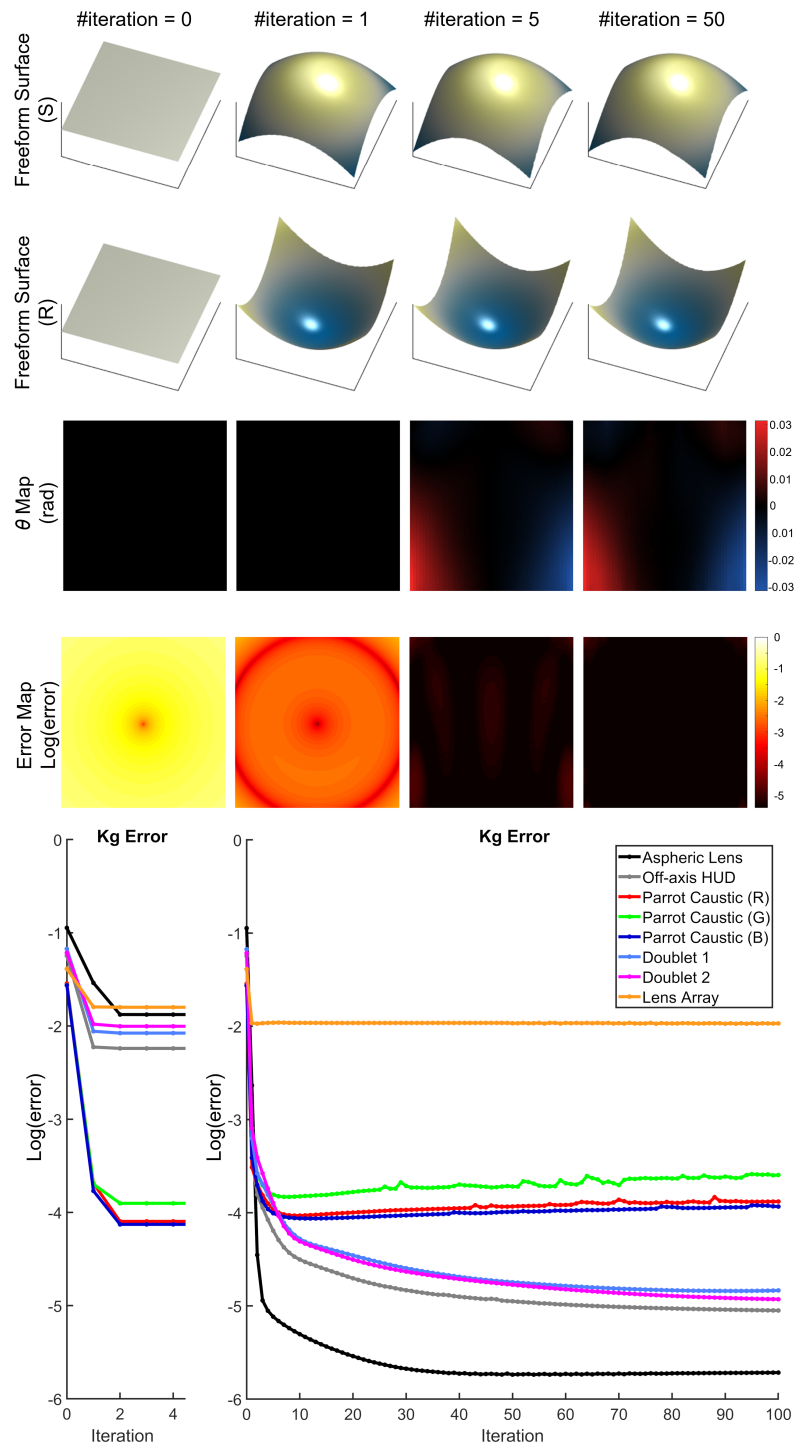

Fig. 6. Optimization results for Section 4.2. Top four rows: optimized surfaces, $\theta$ map, and error plot for the optimization of an aspheric lens HOE. Bottom plot: Iteration errors for different HOEs, with the error definition of Equation 20. Left: If we leave the reference wavefront fixed, the optimization converges rapidly, but the errors remain large. Right: By jointly optimizing both wavefronts through the $\theta$ map, our method yields errors of up to 4 orders of magnitude lower. Each case is also shown in Figure 10.

turning method. Figure 1 (a) shows the recording of an HOE using a pair of our freeform elements, and Figure 11 (b) shows the sample of a full-color caustic parrot HOE.

\subsection{Holographic Printer Bandwidth Optimization}

We designed and built a holographic printer with two wavefront modulation arms using one phase SLM for the signal path and one phase SLM for the reference path. Figure 2 illustrates our holographic printer configuration. An air-bearing mechanical stage (MS) is used to translate the HOE. A laser light source is expanded by a spatial filter (SF) and a lens (L), and two optical paths are combined with a mirror $(M)$ and dichroic mirrors (DMs). The beam is split by a beam splitter (BS), and the SLMs (SANTEC SLM-200) modulate the light in each arm. The wavefronts are de-magnified with an optical relay system including an objective lens (OBL) and filtered by a spatial filter (SF) that blocks the DC frequency and high-order frequencies. Finally, the wavefronts interfere at the HOE plane.

As is common with holographic recording systems [Jeong et al. 2019; Kim et al. 2015; Wakunami et al. 2016], the holographic printer has intrinsic bandwidth limitations. The constraints are visualized in Figure 7. First, the light modulation bandwidth of the system (i.e., the maximum angle at which the beam can be deflected) is limited to $M \arcsin (\lambda / p)$ as indicated by the red square, where $M$ is the de-magnification factor and $p$ is the pixel pitch of the SLM. Also, since we use a phase SLM, the DC noise is filtered out using a spatial filter in the frequency domain of the 4- $f$ system. This spatial filter also blocks some frequencies near the DC component, creating a hole in the middle of the frequency bandwidth domain indicated as a red striped circle. The $f$-number matching of the objective lens in the $4-f$ optics also limits the frequency, indicated as a yellow circle. A double phase encoding is adopted for complex modulation of the wavefront, limiting the frequency bandwidth by half in the x-axis, as indicated by the gray striped region. Furthermore, when the SLM generates a high frequency wavefront, it tends to generate unwanted noise caused by field fringing effects [Fan-Chiang et al. 2006]. In our experience, this noise is significant when the spatial frequency is higher than $50 \%$ of the maximum frequency, and becomes severe near the maximum frequency. Therefore, it is preferable to reduce the frequency band to be as low as possible, i.e., to remain inside the green square.

These constraints can be summarized as two goals. First, we want to avoid the central DC block region to avoid having a hole in the HOE. Second, we aim to reduce the total frequency bandwidth to reduce noise. We formulate these constraints as a cost function to be minimized by the $\theta$ map:

$$
\theta=\underset{\widehat{\theta}}{\operatorname{argmin}}\left\|\overrightarrow{K_{S}}(\widehat{\theta})-\overrightarrow{K_{S 0}}\right\|^{2}+\left\|\overrightarrow{K_{R}}(\widehat{\theta})-\overrightarrow{K_{R 0}}\right\|^{2},
$$

where

$$
\left\|\overrightarrow{K_{S}}(\widehat{\theta})-\overrightarrow{K_{S 0}}\right\|>s_{\mathrm{dc}} \quad \text { and } \quad\left\|\overrightarrow{K_{R}}(\widehat{\theta})-\overrightarrow{K_{R 0}}\right\|>r_{\mathrm{dc}},
$$

where $\overrightarrow{K_{S 0}}$ and $\overrightarrow{K_{R 0}}$ are the $k$-vector of the printer's center bandwidth, which in our case is oblique to the y-axis at 45 degrees for the reference arm and normal to the surface for the signal arm, and $s_{\mathrm{dc}}$ and $r_{\mathrm{dc}}$ are the radii of the DC blocked bandwidth in $k$-space for the reference and signal arms respectively. At $532 \mathrm{~nm}$, our system has a bandwidth of 25 degrees and 12.5 degrees of deflection for the reference arm and signal arms respectively, and 5 degrees and 2.5 degrees respectively for the DC block bandwidth.

Because the printer works with piecewise patches, we do not need to enforce continuity in the $\theta$ map. Equations 21-22 can thus be solved independently as a $1 \mathrm{D}$ optimization for each HOE position. 
(a)

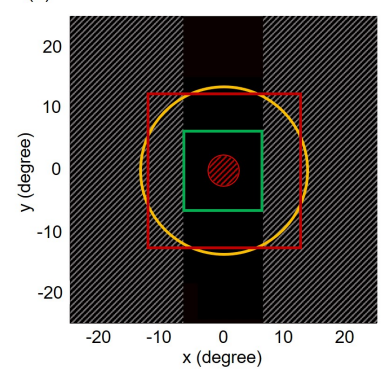

(b)

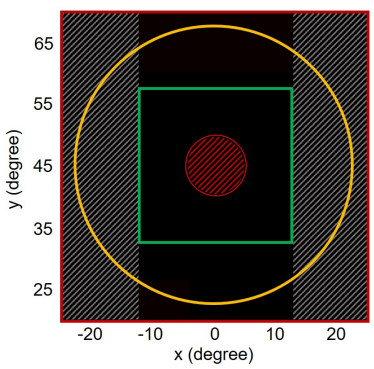

Fig. 7. Bandwidth constraints of the signal arm (a) and reference arm (b) for the holographic printer. The SLM bandwidth constrains the range of possible recording angles (red square), and the DC noise filtering creates a hole in the center of the frequency domain (red striped region). The double phase filtering reduces the bandwidth in one axis by half (gray striped region), and the f-number matching of the objective lens also restricts the bandwidth (yellow circle). The green square shows the preferred bandwidth region to avoid severe noise. When the wavelength changes, the bandwidth values scale proportionally, except for the numerical aperture of the objective lens.

The optimal $\theta$ is either located on the edges of the constraints of Equation 22, or at the minimum of Equation 21, all of which can be solved analytically. The optimal $\theta$ is taken as the argmin of these three branches.

However, because hogels have a finite size, there will likely be discontinuities in the $\theta$ map inside of some of the hogels, where the solution jumps from one branch to the other. In this case, we select the continuous region with the largest area inside the hogel, and use this branch to compute the solution of the whole hogel. This makes each hogel smooth and prevents some high-frequency printing artifacts. Once the $\theta$ map is determined, we compute $\overrightarrow{K_{S}}$ and $\overrightarrow{K_{R}}$ from Equations 15-16, and convert the $k$-vectors to a wavefront by integration [Harker and O'Leary 2008].

Figure 8 shows the examples of optimized $\theta$ map for the HUD and caustic HOEs, with details provided in our supplementary document (Section 2.2). The bandwidth distribution is acquired by calculating the oblique angle in the air from $\overrightarrow{K_{S}}$ and $\overrightarrow{K_{R}}$ over the HOE area and by accumulating them in the frequency domain. For convenience, the bandwidth of the reference and signal arms are plotted in the same domain by normalizing each bandwidth to their own maximum values.

We visualize the bandwidth optimization process in the middle and bottom rows of Figure 8. After optimization, the bandwidth is morphed to avoid the DC block region and moves closer to the lower frequencies. Bandwidth tends to accumulate around the DC block region, as expected from Equation 21. Also, the bandwidth optimization can effectively enhance the total available bandwidth since it enables us to record some frequencies that were initially outside the SLM bandwidth range. For example, in the caustic HOE, $46 \%$ of the frequency spectrum is moved outside of the DC hole, and other higher frequencies are reduced by $36 \%$ (on average) after bandwidth optimization.
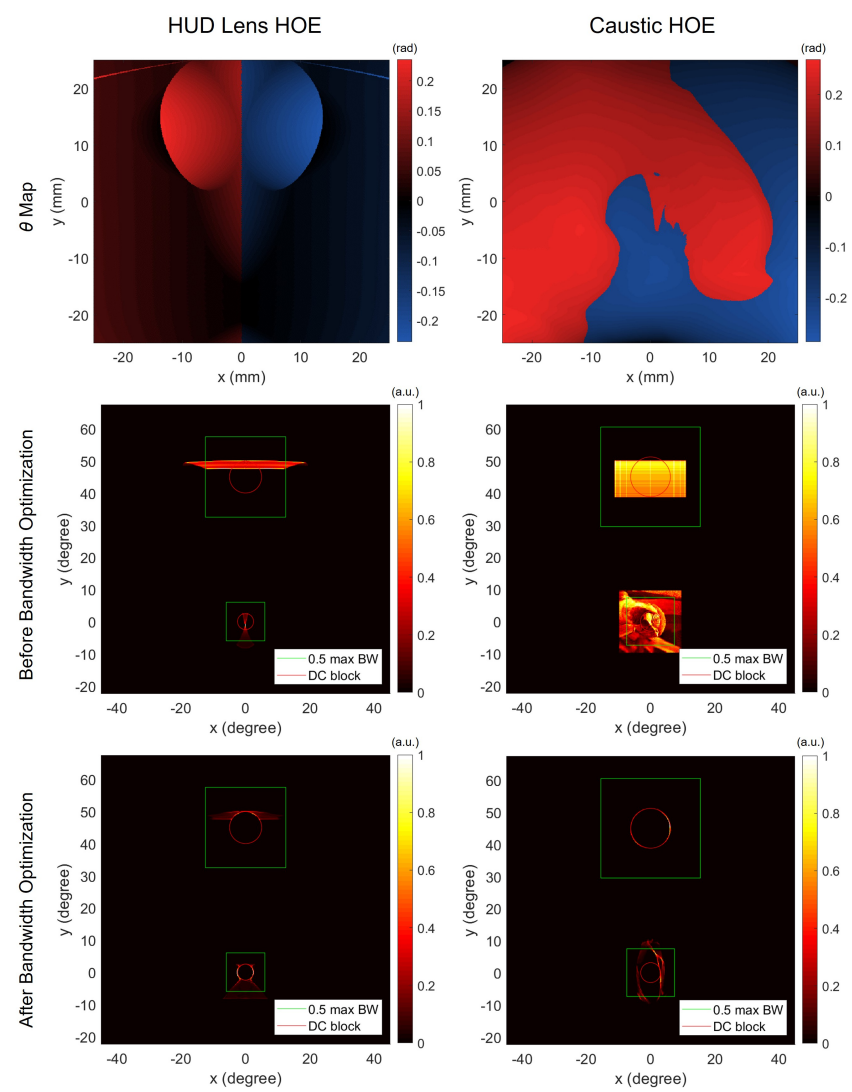

Fig. 8. $\theta$ map (top) and bandwidth distribution, plotted in the frequency domain, before (middle) and after (bottom) the holographic printer optimization. Two different HOE configurations are shown, and the bandwidth of the signal arm and the reference arm are plotted in the same domain to facilitate the presentation.

When using refractive elements (Section 4.2), the phase integration errors accumulate due to the non-integrable nature of the wavefronts. By sectioning the total wavefront into many patches with the holographic printer, this phase integration error can be reduced by orders of magnitude. Figure 9 shows the calculated piecewise wavefronts and shows the reduction of phase integration error as the number of patches increases. The reduction in error does not appear to be affected by the particular HOE profile, and the error always drops below $10^{-4}$ with $50 \times 50$ or more patches, even for the challenging lens-array HOE.

\subsection{Fabrication Details of the Holographic Printer}

The printer has hexagonal shaped hogels with a size of approximately $1.1 \mathrm{~mm}$ by $0.48 \mathrm{~mm}$. Therefore, fabricating a $50 \mathrm{~mm}$ by $50 \mathrm{~mm}$ HOE requires approximately 5,000 hogels. One hogel is recorded in each single exposure, and hogels are stitched together by moving a mechanical stage over the entire HOE area. Our supplementary video shows the printing process in action. The wavefront calculation for hogels is done offline and the exposure of a single hogel only takes 100 milliseconds with an optimized dosage. However, the total 

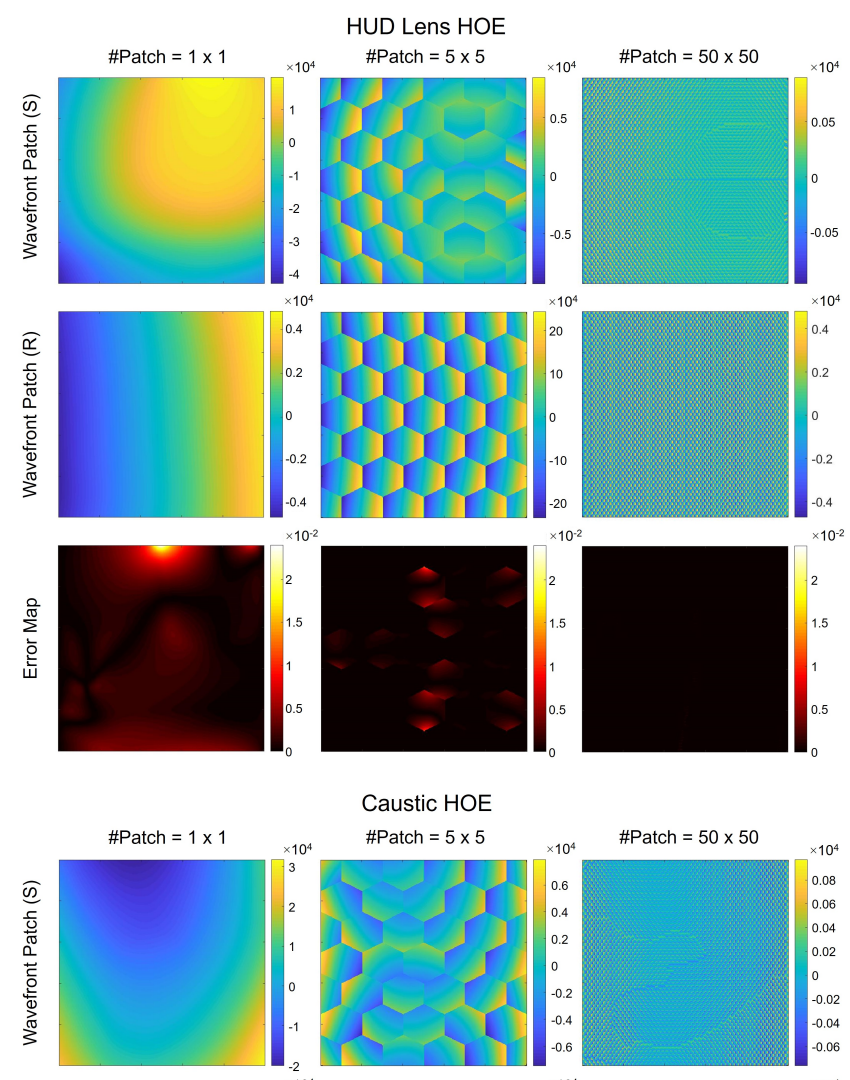

\section{Caustic HOE}
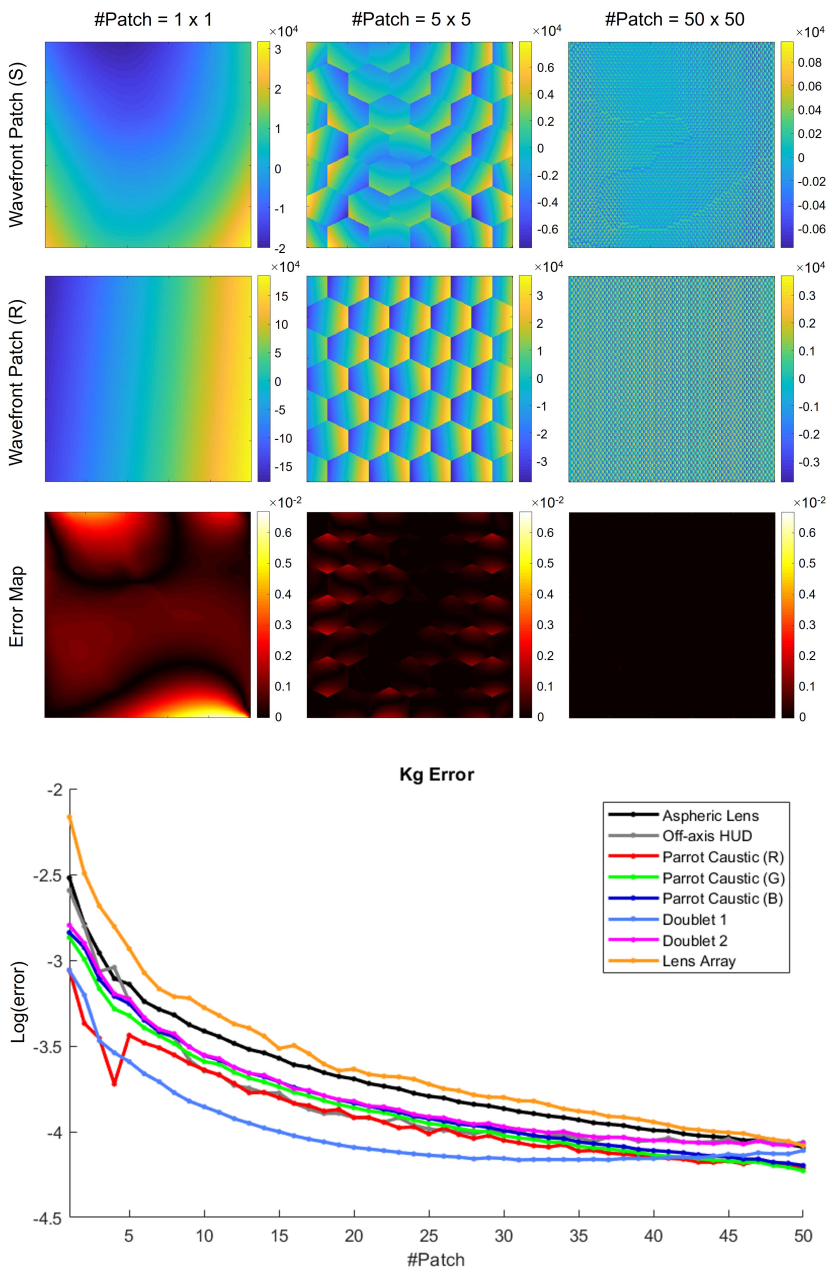

Fig. 9. Computed wavefront patch profiles depending on the number of hogels in the holographic printer. The top two figure groupings show the hogels for two different HOE configurations, and the bottom plot shows the error as a function of the number of hogels for multiple HOEs. fabrication time takes 3 hours to 7 hours due to the time it takes to move and stabilize the mechanical stage. We fabricate and optimize an off-axis HUD and a baseline off-axis HUD for comparison using $532 \mathrm{~nm}$ green laser, while the lens-array HOE and the caustic $\mathrm{HOE}$ are fabricated using $660 \mathrm{~nm}$ red laser (see Figure 12). When changing the wavelength, the system configuration stays the same except for the frequency filter, since the bandwidth scales with the wavelength. Figure 11 (c) shows the holographic printing system and a magnified image of a printed HOE.

\section{RESULTS}

\subsection{Experimental Results}

This section provides the results of our fabricated freeform HOEs. All our HOE configurations are shown in the first row of Figure 10.

For our imaging experiments, we use the same tiger image as in Figure 4, but we do not undistort the displayed images. Figure 12 (a)(c) shows the results of a freeform aspheric HOE: (a) shows a baseline spherical HOE fabricated using a collimated plane wave and a conventional objective lens to generate a spherical conjugate, while (b) and (c) use our optimized diamond-turned elements. In (a), most of the center intensity is lost, and aberrations are seen at the edges. The intensity loss is caused by the shrinkage of the material. Although the HOE is designed to work with a $532 \mathrm{~nm}$ wavelength, the material shrinkage changes its selectivity and it is no longer sensitive to the target wavelength and incidence angle. In contrast, our freeform aspheric HOE is compensated for the shrinkage, therefore (b) shows uniform intensity over the area, while also showing better resolution. (c) shows the result of a full-color freeform aspheric HOE using the wavelength multiplexing of the HOE. This demonstrates that the same pair of freeform elements can be used to record different color channels, at the cost of adding dispersion. Since the freeform surface is optimized for $532 \mathrm{~nm}$, a slight dispersion is observed at the edges, however the full-color image is successfully displayed. In order to achieve full-color achromatic $\mathrm{HOE}$, we could fine-tune the freeform elements to account for refractive index differences and optimize the dosages when recording each color channel.

Figure 12 (d)-(f) shows the results for a singlet HUD. The baseline HOE (d) uses conventional lenses, while (e) and (f) use our optimized diamond-turned elements: (d) shows a typical intensity degradation along the y axis due to shrinkage, while our freeform HOE (e) shows a more uniform intensity. We note here some blurring, especially near the left eye, which is due to a tool mark defect created during our diamond turning process, as shown in the inset. (f) shows a full-color fabrication result using the same diamond-turned element.

Figure $12(\mathrm{~g})$ and $(\mathrm{h})$ uses the holographic printer fabrication method for a HUD lens: (g) prints the baseline hologram without optimization, while $(\mathrm{h})$ is our freeform grating vector profile. In both printed results, the resolution is not as good as with the diamond turning method because the hogel structure limits the numerical aperture, but our optimized HOE (h) shows significantly better intensity uniformity over the field of view than the baseline (g).

Figure 12 (i) shows an imaging application result using a printed freeform lens array HOE (also shown in our supplementary video). At the object position, we put a synthetic eyeball illuminated with a red diode laser light source. Lenslets are optimized to have uniform 

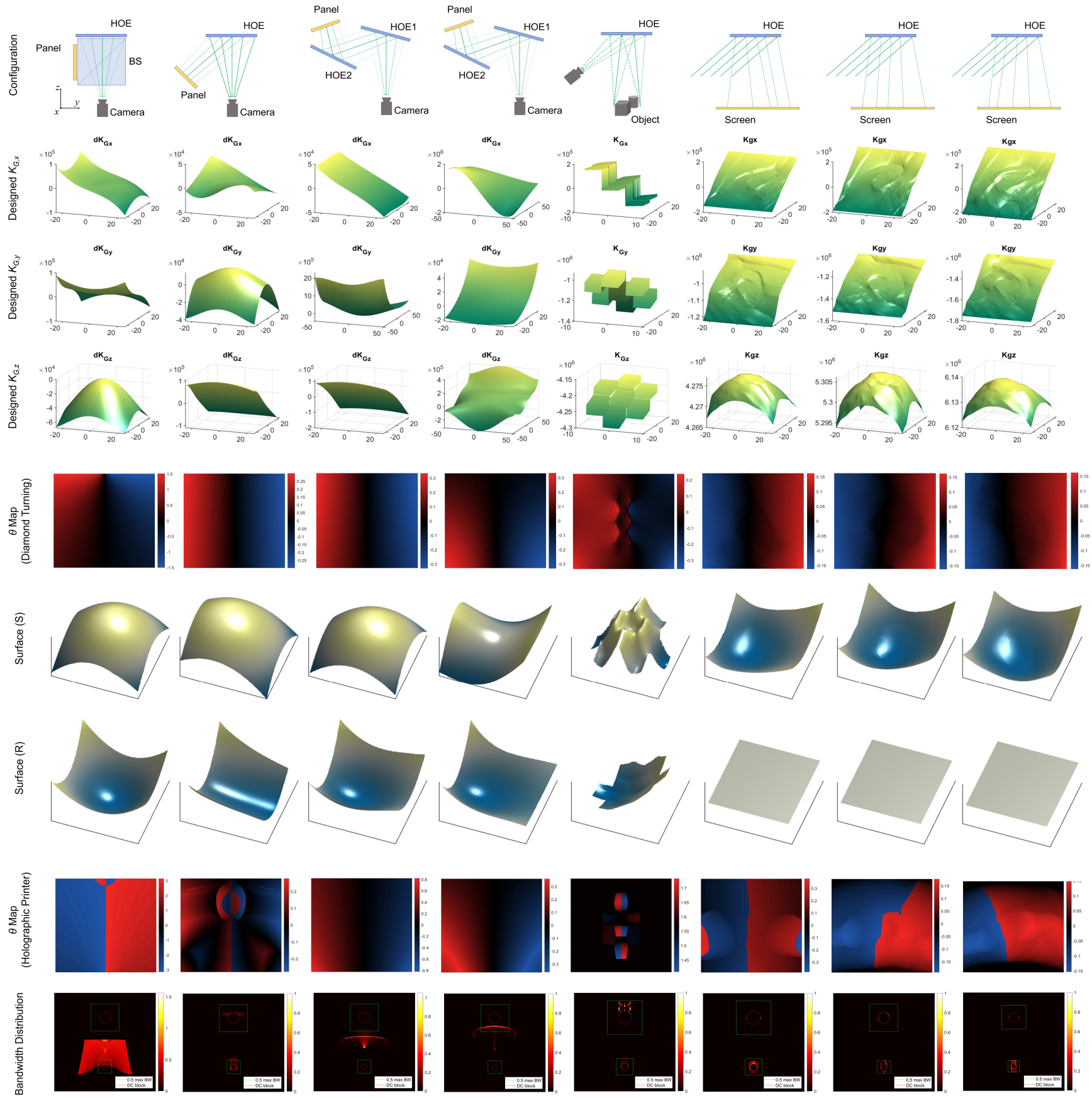

Fig. 10. Simulation results for all the examples used throughout this paper. For each case, we show the HOE configuration (row 1), the target grating vector field components (rows 2-4), the optimized $\theta$ map for the diamond turning method (row 5), the optimized surfaces for the diamond turning method (rows 6-7), the optimized $\theta$ map for the holographic printer (row 8), and the optimized bandwidth distribution for the holographic printer (row 9). 

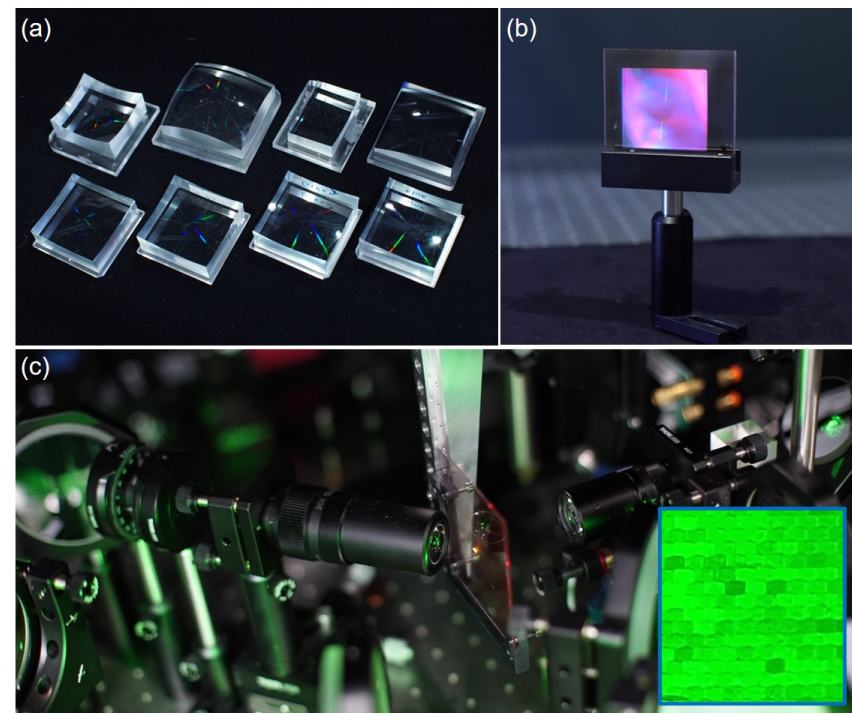

Fig. 11. Photographs of (a) fabricated diamond-turned samples, (b) a fullcolor parrot caustic $\mathrm{HOE}$, and (c) our holographic printing system with two wavefront modulation arms and the magnified image of a printed $\mathrm{HOE}$ showing hogels (inset).

diffraction efficiency over the field of view for the given camera location. Since each lenslet is located at a different position, it provides multi-view images of the eye, which can be used for light field capture and off-axis eye-tracking in VR/AR applications. The holographic printer is well-suited for this example, since the HOE has multiple discrete structures that are difficult to create from smooth diamond-turned glass profiles.

In Figure 12 (j)-(l), we demonstrate the first full-color caustic HOE. Thanks to the multiplexability and high selectivity of HOEs, it is possible to project a full color caustic image either by wavelength multiplexing or by stacking red, green, and blue HOEs. Here, we used the latter approach as it creates a higher diffraction efficiency. The parrot caustic HOE is fabricated using the diamond turning method, and the image is projected with different types of illumination: (j) a coherent laser light source, (k) a laser with reduced spatial coherency using a de-speckler, and (l) a broadband incoherent light source using a white LED collimated with a $150 \mathrm{~mm}$ lens. The best color contrast is obtained from the coherent light illumination, and the resolution and contrast of the caustic image degrade as the coherency is reduced. However, the image is still definitely visible even with the broadband light source. The target parrot image is presented in the supplementary document (Section 2.2).

\subsection{Limitations and Future Work}

Curl-free Decomposition. Our optimization method for the diamond turning approach (Algorithm 1) could potentially be improved to more explicitly take into account the integrability of the surface normals. In general, a normal vector field defined on a flat domain can be reconstructed as a height profile if and only if it generates a curl-free tangential field, but this condition is more complex if the normal field is defined on the arbitrarily-shaped glass surface. Still, future work could include a better projection operator (similar to a Helmholtz decomposition) that can better fit our framework. Although using this improved iterative process may lead to the same optimal solutions, it may converge faster and in a more stable manner, which could enable the optimization and fabrication of even more complex HOEs.

Limitations of the Holographic Printer. The air-bearing stage of the holographic printer generates vibrations which sometimes prevent the proper grating formation in the hogels, as seen by the darker hogels in the inset of Figure 11 (c). We expect a piezo stage can be used to reduce vibrations in the system. In addition, the fundamental bandwidth of the system is limited by the spatial light modulator. By increasing the de-magnification factor $M$, the deflection angle range can be increased, but at the cost of reducing the maximum hogel size by a factor of $M$. A smaller hogel size would cause larger diffraction, as well as increase fabrication time. By decreasing $M$, the maximum deflection angle is reduced which limits the versatility of designs. Despite these limitations, the holographic printer provides an efficient way to quickly prototype HOE designs. With a large enough bandwidth, we expect that the capability of the printer can expand to record poly-chromatic HOEs at a single wavelength. For example, a holographic printer working with ultraviolet laser immersed in an index-matched medium can in theory achieve enough bandwidth to record any poly-chromatic $\mathrm{HOE}$ in the visible wavelengths.

HOEs with freeform geometries. In this work, we limited our design of freeform volume grating profiles to $2 \mathrm{D}$ planes rather than $3 \mathrm{D}$ freeform surfaces. For some VR/AR applications, it could be beneficial to create HOEs with physically curved form-factors, for example, for HOEs laminated on curved windshields or glasses [Bang et al. 2019; Blanche et al. 2019]. We expect our fabrication framework to expand well to such cases, since neither the printer or the diamond turning approaches require the $\mathrm{HOE}$ to be flat, and the optimization method of Algorithm 1 could be adapted to intersect rays with a curved surface on step 3. Optimizing the shape of the HOE as part of our method would provide us with more degrees of freedom and would broaden applications, but we leave this as future work.

\section{CONCLUSION}

We presented a pipeline for the design, optimization, and fabrication of freeform holographic optical elements. Our framework characterizes the volume grating of HOEs as a $k$-vector field, which enables a tractable optimization method based on ray tracing and the explicit tuning of HOE selectivity. To support practical applications, we have provided two methods to fabricate these optimized freeform HOEs. With the first approach, we have developed a novel iterative method to generate high-quality, diamond-turned freeform refractive surfaces to shape the reference and signal wavefronts. With the second approach, we have built a two-arms holographic printer and proposed a bandwidth optimization scheme to find suitable recording wavefronts with limited printer bandwidth. As a result, we have demonstrated a broad set of design and fabrication examples, including aspheric lenses, HUDs, doublet lenses, and lens arrays HOEs, as well as the first full-color caustic projection HOE. 


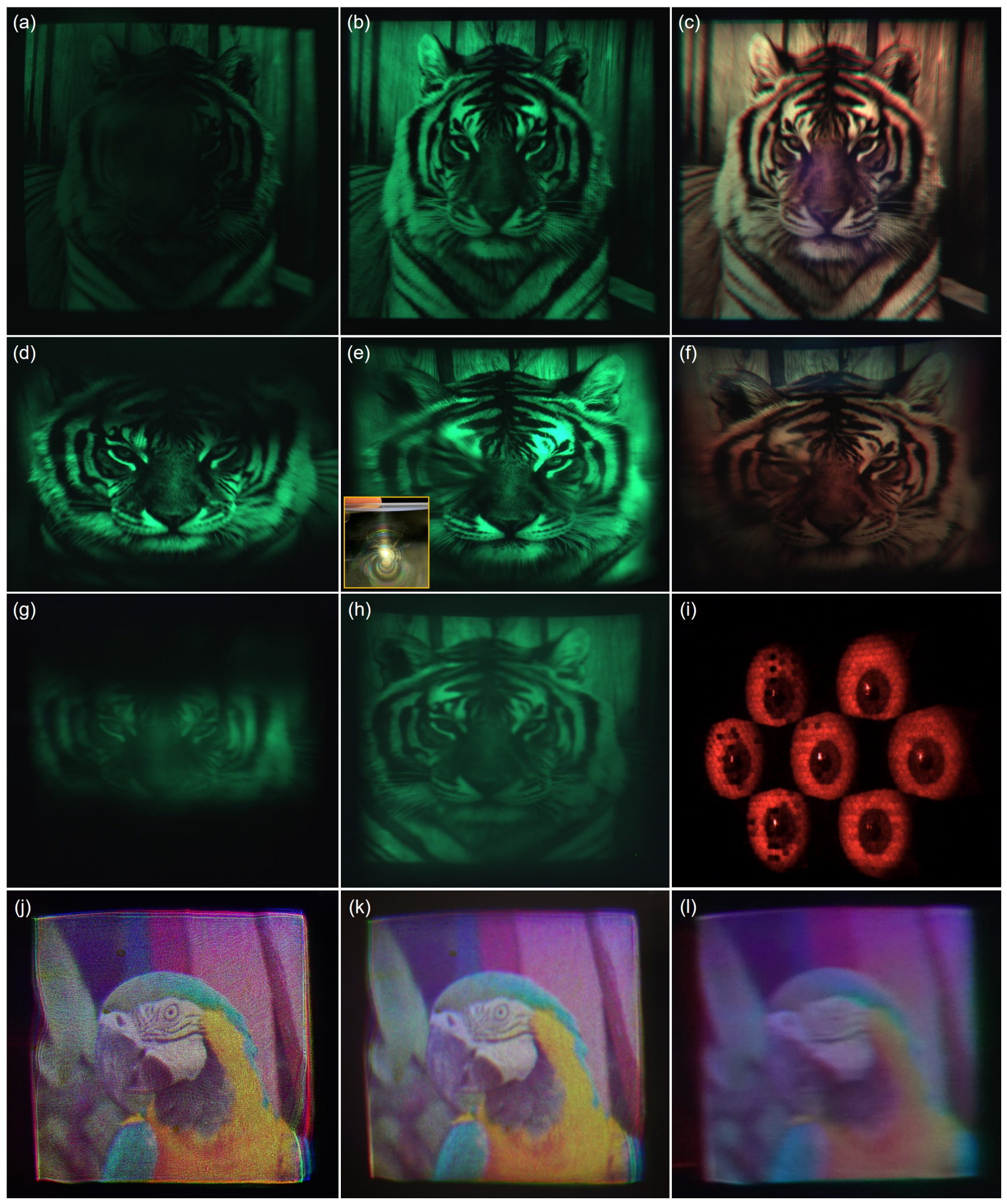

Fig. 12. Photographs of experimental results for our freeform HOEs. (a)-(c): an aspheric lens HOE. (d)-(f): a HUD lens HOE. (g) and (h): a printed HUD lens HOE. (i): a printed lens array HOE. (j)-(l): caustic HOEs. See Section 5.1 for a detailed description of each result.

ACM Trans. Graph., Vol. 39, No. 6, Article 184. Publication date: December 2020. 


\section{ACKNOWLEDGMENTS}

The parrot image (by Siripong Jitchum) and tiger image (by LMPphoto) are licensed from Shutterstock. We thank Andrew Maimone for useful discussions and advice, and Alexander Sohn and Neil Naples for their help with diamond turning.

\section{REFERENCES}

Kaan Akşit, Ward Lopes, Jonghyun Kim, Peter Shirley, and David Luebke. 2017. NearEye Varifocal Augmented Reality Display Using See-through Screens. ACM Trans Graph. 36, 6, Article 189 (Nov. 2017), 13 pages.

Kaan Akșit, Praneeth Chakravarthula, Kishore Rathinavel, Youngmo Jeong, Rachel Albert, Henry Fuchs, and David Luebke. 2019. Manufacturing application-driven foveated near-eye displays. IEEE Transactions on Visualization and Computer Graphics (2019).

Y. Amitai, A. A. Friesem, and V. Weiss. 1990. Designing holographic lenses with different recording and readout wavelengths. F. Opt. Soc. Am. A 7, 1 (Jan 1990), 80-86.

Kiseung Bang, Changwon Jang, and Byoungho Lee. 2019. Curved holographic optical elements and applications for curved see-through displays. Fournal of Information Display (02 2019), 1-15.

Aaron Bauer, Eric Schiesser, and Jannick Rolland. 2018. Starting geometry creation and design method for freeform optics. Nature Communications 9 (12 2018).

Pierre-Alexandre Blanche, Colton Bigler, Craig Draper, and Micah Mann. 2019. Holographic See-Through Displays. In Digital Holography and Three-Dimensional Imaging 2019. Optical Society of America, M3A.1.

Richard Byrd, Jean Charles Gilbert, and Jorge Nocedal. 2000. A trust region method based on interior point techniques for nonlinear programming. Mathematical Programming 89 (11 2000), 149-185.

D. H. Close. 1975. Holographic optical elements. Opt. Eng. 14, 5 (1975), 145402

H. Coufal, D. Psaltis, and G.T. Sincerbox. 2000. Holographic data storage. Springer.

Gerwin Damberg and Wolfgang Heidrich. 2015. Efficient freeform lens optimization for computational caustic displays. Opt. Express 23, 8 (Apr 2015), 10224-10232.

R. C. Fairchild and J. R. Fienup. 1982. Computer-originated aspheric holographic optical elements. Optical Engineering 21, 1 (1982), 133 - 140.

Kuan-Hsu Fan-Chiang, Shin-Tson Wu, and Shu-Hsia Chen. 2006. Fringing-field effects on high-resolution liquid crystal microdisplays. Journal of Display Technology (2006).

Fengzhou Fang, Ying Cheng, and Xiaodong Zhang. 2013. Design of freeform optics. Advanced Optical Technologies 2 (12 2013), 445-453.

Kenneth Garrard, Thomas Bruegge, Jeff Hoffman, Thomas Dow, and Alex Sohn. 2005. Design tools for freeform optics. Proc. SPIE 5874 (08 2005).

Ralf Haeussler, Y. Gritsai, E. Zschau, R. Missbach, H. Sahm, M. Stock, and H. Stolle 2017. Large real-time holographic 3D displays: Enabling components and results Applied Optics 56 (05 2017), F45.

Matthew Harker and Paul O'Leary. 2008. Least squares surface reconstruction from measured gradient fields. Proceedings / CVPR, IEEE Computer Society Conference on Computer Vision and Pattern Recognition, 1 - 7.

Johannes Hofmann, Anna-Katharina Friedel, Reinhold Fiess, and Wilhelm Stork. 2019 Angle-compensated holographic wave front printing for the fabrication of holographic optical elements operating in the infrared. Optical Engineering 59 (2019).

Keehoon Hong, Jiwoon Yeom, Changwon Jang, Jisoo Hong, and Byoungho Lee. 2014 Full-color lens-array holographic optical element for three-dimensional optical see-through augmented reality. Optics letters 39 (01 2014), 127-30.

Boaz Jessie Jackin, Lode Jorissen, Ryutaro Oi, Jui Yi Wu, Koki Wakunami, Makoto Okui, Yasuyuki Ichihashi, Philippe Bekaert, Yi Pai Huang, and Kenji Yamamoto. 2018 Digitally designed holographic optical element for light field displays. Opt. Lett. 43, 15 (Aug 2018), 3738-3741.

Changwon Jang, Kiseung Bang, Gang Li, and Byoungho Lee. 2018. Holographic near-eye display with expanded eye-box. ACM Transactions on Graphics 37 (2018), 1-14.

Changwon Jang, Kiseung Bang, Seokil Moon, Jonghyun Kim, Seungjae Lee, and By oungho Lee. 2017. Retinal 3D: augmented reality near-eye display via pupil-tracked light field projection on retina. ACM Transactions on Graphics 36, 6 (2017), 190.

Daniel Jeon, Seung-Hwan Baek, Shinyoung Yi, Qiang Fu, Xiong Dun, Wolfgang Heidrich, and Min Kim. 2019. Compact snapshot hyperspectral imaging with diffracted rotation. ACM Transactions on Graphics 38 (07 2019), 1-13.

Jinsoo Jeong, Jaebum Cho, Changwon Jang, Chanhyung Yoo, and Byoungho Lee. 2019 Design and fabrication of extended eye-box holographic lens using holographic printer. In Digital Holography and Three-Dimensional Imaging 2019. OSA, W2A.3.

J. Jeong, C. Lee, B. Lee, S. Lee, S. Moon, G. Sung, H. Lee, and B. Lee. 2020. Holographically Printed Freeform Mirror Array for Augmented Reality Near-Eye Display. IEEE Photonics Technology Letters 32, 16 (2020), 991-994.

Seyedmahdi Kazempourradi, Erdem Ulusoy, and Hakan Urey. 2019. Full-color computational holographic near-eye display. Fournal of Information Display 20, 2 (2019).
Jonghyun Kim, Zander Majercik, Peter Shirley, Josef Spjut, Morgan McGuire, David Luebke, Youngmo Jeong, Michael Stengel, Kaan Akşit, Rachel Albert, Ben Boudaoud Trey Greer, Joohwan Kim, and Ward Lopes. 2019. Foveated AR: dynamically-foveated augmented reality display. ACM Transactions on Graphics 38 (07 2019), 1-15.

Youngmin Kim, Elena Stoykova, Hoonjong Kang, Sunghee Hong, Joosup Park, Jiyong Park, and Jisoo Hong. 2015. Seamless full color holographic printing method based on spatial partitioning of SLM. Opt. Express 23, 1 (Jan 2015), 172-182.

H. Kogelnik. 1969. Coupled wave theory for thick hologram gratings. The Bell System Technical fournal 48, 9 (Nov 1969), 2909-2947.

George Koulieris, Kaan Akșit, M. Stengel, R. Mantiuk, Katerina Mania, and C. Richardt. 2019. Near-eye display and tracking technologies for virtual and augmented reality. Computer Graphics Forum 38 (05 2019), 493-519.

Seungjae Lee, Changwon Jang, Seokil Moon, Jaebum Cho, and Byoungho Lee. 2016a Additive light field displays: realization of augmented reality with holographic optical elements. ACM Transactions on Graphics 35 (07 2016), 1-13.

Seungjae Lee, Byounghyo Lee, Jaebum Cho, Changwon Jang, Jonghyun Kim, and Byoungho Lee. 2016b. Analysis and implementation of hologram lenses for seethrough head-mounted display. IEEE Photonics Technology Letters PP (2016).

Changgeng Liu, Juan Liu, Lei Liu, Xincheng Yao, and Beatrice Pazzucconi. 2018. A holographic waveguide based eye tracker. Proceedings of SPIE-the International Society for Optical Engineering 10474, 65.

Andrew Maimone, Andreas Georgiou, and Joel Kollin. 2017. Holographic near-eye displays for virtual and augmented reality. ACM Transactions on Graphics (2017).

Andrew Maimone and Junren Wang. 2020. Holographic optics for thin and. lightweight virtual reality. ACM Trans. Graph. 39, 4 (Jul 2020).

Quentin Mérigot, Jocelyn Meyron, and Boris Thibert. 2017. Light in Power: a general and parameter-free algorithm for caustic design. (08 2017).

Hiroshi Mukawa, Katsuyuki Akutsu, Ikuo Matsumura, Satoshi Nakano, Takuji Yoshida, Mieko Kuwahara, Kazuma Aiki, and Masataka Ogawa. 2008. A full Color eyewear display Using holographic planar waveguides. Sid Symposium Digest of Technical Papers 39 (05 2008).

Eduard R Muslimov, Marc Ferrari, Emmanuel Hugot, Jean-Claude Bouret, Coralie Neiner, Simona Lombardo, Gerard R Lemaitre, Robert Grange, and Ilia A Guskov. 2018. Design and modeling of spectrographs with holographic gratings on freeform surfaces. Optical Engineering 57, 12 (2018), 125105.

Takashi Oku, Katsuyuki Akutsu, Mieko Kuwahara, Takuji Yoshida, Eisaku Kato, Kazuma Aiki, Ikuo Matsumura, Satoshi Nakano, Akio Machida, and Hiroshi Mukawa. 2015. 15.2: High-luminance see-through eyewear display with novel volume hologram waveguide technology. SID Symposium Digest of Technical Papers 46 (06 2015).

Haichao Peng, Dewen Cheng, Jian Han, Chen Xu, Weitao Song, Liuzhu Ha, Jian Yang, Quanxing Hu, and Yongtian Wang. 2014. Design and fabrication of a holographic head-up display with asymmetric field of view. Applied Optics 53 (10 2014).

Yifan Peng, Xiong Dun, Qilin Sun, and Wolfgang Heidrich. 2017. Mix-and-match holography. ACM Transactions on Graphics 36 (11 2017), 1-12.

Yifan Peng, Qilin Sun, Xiong Dun, Gordon Wetzstein, Wolfgang Heidrich, and Felix Heide. 2019. Learned large field-of-view imaging with thin-plate optics. $A C M$ Transactions on Graphics 38 (11 2019), 1-14.

Bahaa E A Saleh and Malvin Carl Teich. 2007. Fundamentals of photonics; 2nd ed. Wiley, New York, NY.

Yuliy Schwartzburg, Romain Testuz, Andrea Tagliasacchi, and Mark Pauly. 2014. Highcontrast computational caustic design. ACM Transactions on Graphics 33 (07 2014).

Hua Shen, Rihong Zhu, Zhishan Gao, E. Y. B. PUN, W. H. Wong, and Xiaoli Zhu. 2013. Design and fabrication of computer-generated holograms for testing optical freeformsurfaces. Chin. Opt. Lett. 11, 3 (Mar 2013), 032201.

Vincent Sitzmann, Steven Diamond, Yifan Peng, Xiong Dun, Stephen Boyd, Wolfgang Heidrich, Felix Heide, and Gordon Wetzstein. 2018. End-to-end optimization of optics and image processing for achromatic extended depth of field and super-resolution imaging. ACM Transactions on Graphics 37 (07 2018), 1-13.

Synopsys. 2020. CodeV. https://www.synopsys.com/optical-solutions/codev.html

Koki Wakunami, Po-Yuan Hsieh, Ryutaro Oi, Takanori Senoh, Hisayuki Sasaki, Yasuyuki Ichihashi, Makoto Okui, Yi-Pai Huang, and Kenji Yamamoto. 2016. Projection-type see-through holographic three-dimensional display. Nature Communications 7 (2016).

Patrick Wissmann, Se Baek Oh, and George Barbastathis. 2008. Simulation and optimization of volume holographic imaging systems in Zemax ${ }^{\circledR}$. Optics express 16 (06 2008), 7516-24.

Amnon Yariv and Pochi Yeh. 1983. Optical waves in crystals: propagation and control of laser radiation.

Genzhi Ye, Sundeep Jolly, V. Michael Bove Jr, Qionghai Dai, Ramesh Raskar, and Gordon Wetzstein. 2014. Toward BxDF display using multilayer diffraction. ACM Transactions on Graphics 33 (11 2014), 1-14.

Yonghao Yue, Kei Iwasaki, Bing-Yu Chen, Yoshinori Dobashi, and Tomoyuki Nishita 2014. Poisson-based continuous surface generation for goal-based caustics. ACM Transactions on Graphics (TOG) 33, 3 (2014), 31.

Zebra Imaging. 2020. Zebra Imaging. http://www.zebraimaging.com/

Zemax. 2020. Optics Studio. https://www.zemax.com/ 